\title{
RATES AND PROPERTIES OF TYPE Ia SUPERNOVAE AS A FUNCTION OF MASS AND STAR FORMATION IN THEIR HOST GALAXIES
}

\author{
M. Sullivan, ${ }^{1}$ D. Le Borgne ${ }^{2}$ C. J. Pritchet, ${ }^{3}$ A. Hodsman, ${ }^{1}$ J. D. Neill, ${ }^{3}$ D. A. Howell, ${ }^{1}$ R. G. Carlberg, ${ }^{1}$ \\ P. Astier, ${ }^{4}$ E. Aubourg, ${ }^{2,5}$ D. Balam, ${ }^{3}$ S. Basa, ${ }^{6}$ A. Conley, ${ }^{1}$ S. Fabbro, ${ }^{7}$ D. Fouchez, ${ }^{8}$ J. Guy, ${ }^{4}$ \\ I. Hook,${ }^{9}$ R. Pain, ${ }^{4}$ N. Palanque-Delabrouille, ${ }^{2}$ K. Perrett, ${ }^{1}$ N. Regnault, ${ }^{4}$ J. Rich, ${ }^{2}$ \\ R. Taillet, ${ }^{4,10}$ S. Baumont, ${ }^{4}$ J. Bronder, ${ }^{9}$ R. S. Ellis, ${ }^{11}$ M. Filiol ${ }^{6}$ V. Lusset, ${ }^{2}$ \\ S. Perlmutter, ${ }^{12}$ P. Ripoche, ${ }^{8}$ and C. TaO ${ }^{8}$ \\ Received 2006 February 6; accepted 2006 May 17
}

\begin{abstract}
We show that Type Ia supernovae (SNe Ia) are formed within both very young and old stellar populations, with observed rates that depend on the stellar mass and mean star formation rates (SFRs) of their host galaxies. Models in which the SN Ia rate depends solely on host galaxy stellar mass are ruled out with $>99 \%$ confidence. Our analysis is based on 100 spectroscopically confirmed SNe Ia, plus 24 photometrically classified events, all from the Supernova Legacy Survey (SNLS) and distributed over $0.2<z<0.75$. We estimate stellar masses and SFRs for the SN Ia host galaxies by fitting their broadband spectral energy distributions with the galaxy spectral synthesis code PÉGASE.2. We show that the SN Ia rate per unit mass is proportional to the specific SFR of the parent galaxies-more vigorously star-forming galaxies host more SNe Ia per unit stellar mass, broadly equivalent to the trend of increasing SN Ia rate in later type galaxies seen in the local universe. Following earlier suggestions for a simple "two-component" model approximating the SN Ia rate, we find bivariate linear dependencies of the SN Ia rate on both the stellar masses and the mean SFRs of the host systems. We find that the SN Ia rate can be well represented as the sum of $5.3 \pm 1.1 \times 10^{-14} \mathrm{SNe}$ $\mathrm{yr}^{-1} M_{\odot}^{-1}$ and $3.9 \pm 0.7 \times 10^{-4} \mathrm{SNe} \mathrm{yr}^{-1}\left(M_{\odot} \mathrm{yr}^{-1}\right)^{-1}$ of star formation. We also demonstrate a dependence of distant SN Ia light-curve shapes on star formation in the host galaxy, similar to trends observed locally. Passive galaxies, with no star formation, preferentially host faster declining/dimmer SNe Ia, while brighter events are found in systems with ongoing star formation.
\end{abstract}

Subject headings: distance scale — galaxies: evolution — supernovae: general — surveys

Online material: color figures

\section{INTRODUCTION}

Type Ia supernovae (SNe Ia) represent cosmologists' most direct probe of the cosmic expansion history, yet an understanding of the composition of their progenitor systems has not yet been achieved (e.g., Hillebrandt \& Niemeyer 2000). In principle, this uncertainty can be reduced and constraints placed on the nature of the progenitor if the typical explosion timescale of SNe Ia can be determined. This "delay time" parameterizes the distribution of times between the binary system formation and subsequent

\footnotetext{
${ }^{1}$ Department of Astronomy and Astrophysics, University of Toronto, 60 St. George Street, Toronto, ON M5S 3H8, Canada.

2 DAPNIA/Service d'Astrophysique, CEA-Saclay, 91191 Gif-sur-Yvette Cedex, France.

3 Department of Physics and Astronomy, University of Victoria, P.O. Box 3055, Victoria, BC V8W 3P6, Canada.

${ }^{4}$ Laboratoire de Physique Nucleaire et de Haute Energies de Paris (LPNHE), CNRS-IN2P3; and University of Paris VI and VII, 75005 Paris, France.

5 APC, 11 Place Marcelin Berthelot, 75231 Paris Cedex 5, France.

${ }^{6}$ Laboratoire Astrophysique de Marseille (LAM), CNRS, BP8, Traverse du Siphon, 13376 Marseille Cedex 12, France.

${ }^{7}$ Centro Multidisciplinar de Astrofísica (CENTRA), Instituto Superior Técnico, Avenida Rovisco Pais, 1049 Lisbon, Portugal.

${ }^{8}$ Centre de Physique des Particules de Marseille (CPPM), CNRS-IN2P3; and University Aix Marseille II, Case 907, 13288 Marseille Cedex 9, France.

9 Department of Astrophysics, University of Oxford, Denys Wilkinson Building, Keble Road, Oxford OX1 3RH, UK.

${ }_{10}$ Université de Savoie, 73000 Chambéry, France.

${ }^{11}$ California Institute of Technology, 1200 East California Boulevard, Pasadena, CA 91125.

${ }_{12}$ Lawrence Berkeley National Laboratory, 1 Cyclotron Road, Berkeley, CA 94720 .
}

SN explosion following accretion of material from a secondary companion (e.g., Madau et al. 1998a; see Greggio [2005] for a comprehensive analysis of rate model formalizations).

Various pieces of observational evidence have been used to place different constraints on the value of this delay time. The principal approach compares observed SN Ia rates with a predicted rate generated by convolving a delay function with an assumed cosmic star formation history (e.g., Madau et al. 1998a; Gal-Yam $\&$ Maoz 2004). Studies using this approach have determined a wide range of delay times: $\simeq 2-4$ Gyr (Strolger et al. 2004, 2005), $\geq 2$ Gyr (Gal-Yam \& Maoz 2004), and $\leq 1$ Gyr (Barris \& Tonry 2006).

A second technique uses comparisons of the SN Ia rate in $z<1$ galaxy clusters with the observed cluster iron content. If $\mathrm{SNe} \mathrm{Ia}$ are assumed to represent the dominant source of iron in clusters, the low cluster SN Ia rate at low redshift implies much of the iron must have been produced from events at higher redshift - and hence suggests delay times of $<2$ Gyr (Maoz \& Gal-Yam 2004). The third piece of evidence follows from the observation that SNe Ia are substantially more common in star-forming later type galaxies than in early-type systems (e.g., Oemler \& Tinsley 1979). The rate per unit mass is significantly higher both in later type galaxies than in E/S0 systems (van den Bergh 1990; Della Valle \& Livio 1994; Mannucci et al. 2005) and in bluer galaxies than in red galaxies (Mannucci et al. 2005), with an enhancement of SNe Ia in early-type galaxies that are radio loud (Della Valle et al. 2005). Furthermore, within star-forming galaxies, SNe Ia are rarer in galaxy bulges than in their disks (Wang et al. 1997). This suggests some dependence of the SN Ia rate on recent star formation (and 
hence very short delay times). Finally, there is the observation that $\mathrm{SNe}$ Ia are common in old evolved systems with little recent star formation activity (Cappellaro et al. 1999; Mannucci et al. 2005), suggesting some progenitors have delay times of at least several gigayears; other studies of the star formation histories of local SN Ia host galaxies claim a delay time lower limit of $\sim 2 \mathrm{Gyr}$ (Gallagher et al. 2005).

These contradictions can be resolved by removing the constraint of a single delay time parameterizing all SN Ia explosions and instead using a "two-component" distribution (or even a more general function), similar to the models proposed by Mannucci et al. (2005, 2006) and Scannapieco \& Bildsten (2005, hereafter SB05). Such models comprise a "prompt" (or small delay time) SN Ia component, essentially dependent on recent star formation, and an "old" (larger delay time) component, dependent on the number of lower mass stars. The total rate of SNe Ia is then a combination of these different functions. These scenarios are able to resolve many of the observational contradictions described above (see discussion in SB05).

These two-component models have implicit but important implications for the progenitors of SNe Ia that might impact their use as calibrated standard candles to derive cosmological parameters (Riess et al. 1998; Perlmutter et al. 1999; Astier et al. 2006). The possibility of subtle differences between SNe Ia from the two components, or a change in the relative fraction of the two types with redshift, are of potential concern. As such, it is vital to test and parameterize the SN Ia models in as many ways as possible. One way to do this is to look at the rates and properties of $\mathrm{SNe}$ Ia in relation to the environments or galaxies in which they explode. Galaxies across the Hubble sequence provide an ideal laboratory for studying SNe Ia due the range of potential progenitor stellar populations that can be probed, from starburst galaxies with dominant young populations of stars, through normal galaxies such as the Milky Way with a substantial fraction of evolved stellar mass, to the old, evolved elliptical galaxies in massive galaxy clusters that are essentially comprised of homogeneous old stellar populations.

In this paper, we examine the properties of high-redshift SN Ia host galaxies and calculate the frequency of occurrence of SNe Ia in galaxies of different type, exploring the parameters governing the $\mathrm{SN}$ Ia rate. Our motivation is to measure the SN Ia rate as a function of the stellar mass and star formation rates (SFRs) of the host galaxies in order to test the various predictions of the models described above. Performing these tests requires not only a large, homogeneous data set of SNe Ia with well-understood detection efficiency characteristics, but additionally a multiwavelength data set for their host galaxy systems that can be used to constrain their stellar populations. The intermediate-redshift Supernova Legacy Survey (SNLS) satisfies both requirements.

The SNLS uses repeat $u^{*} g^{\prime} r^{\prime} i^{\prime} z^{\prime}$ imaging of four square degree fields to conduct a "rolling" high-redshift SN search. The repeat imaging allows not only the construction of high-quality multiband $g^{\prime} r^{\prime} i^{\prime} z^{\prime}$ SN light curves (Astier et al. 2006), but also extremely deep images of the survey fields, and hence precise information on the broadband spectral energy distributions (SEDs) of the SN host galaxies can be collected. Perhaps surprisingly, precision work on $\mathrm{SNe}$ Ia rates is most efficiently performed at these intermediate redshifts $(z \sim 0.5)$; low-redshift SN Ia rates are still far less precise than those at higher redshift (e.g., Neill et al. 2006) due to the homogeneity of high-redshift rolling SN searches.

A plan of the paper follows. Sections 2, 3, and 4 detail the framework on which our subsequent analyses in $\S \S 5$ and 6 are built. In $\S 2$ we introduce the SNLS and discuss the construction of deep optical stacks of the survey field as well as the host gal- axy identification and flux measurement. Section 3 describes the galaxy SED fitting, and $\S 4$ details the various incompleteness corrections we apply to our galaxy and SN Ia samples. Our analysis is contained in $\S \S 5$ and 6 . In $\S 5$ we examine the rate of SNe Ia as a function of various host galaxy parameters, and in $\S 6$ we examine the impact of environment on SN Ia light-curve shape parameters. We summarize in $\S 7$.

Throughout the paper, we assume a cosmology of $\Omega_{M}=0.3$, $\Omega_{\Lambda}=0.7, H_{0}=70 \mathrm{~km} \mathrm{~s}^{-1} \mathrm{Mpc}^{-1}$, and use the $\mathrm{AB}$ photometric system (Oke \& Gunn 1983).

\section{THE SNLS DATA SET}

This section describes the observational data set that we use for our analysis in this paper. We first describe the data set of $\mathrm{SNe}$ Ia, and then the data sets of both the SN Ia host galaxies and the general galaxy population.

\subsection{Supernova Data}

Our SN Ia data come from the SNLS. The SNLS is a rolling search for distant SNe with a primary science goal of using 700 high-redshift SNe Ia to determine the average equation-of-state parameter of dark energy, $\langle w\rangle$ (see Astier et al. 2006). SNLS exploits the square degree MegaCam camera (Boulade et al. 2003) on the Canada-France-Hawaii Telescope (CFHT) to conduct repeat $g^{\prime} r^{\prime} i^{\prime} z^{\prime}$ imaging of four low Galactic extinction fields (named D1-D4; see Sullivan et al. [2006] for the field coordinates), imaged as part of the "deep" component of the 5 year CFHT Legacy Survey (CFHTLS). The data are time sequenced with observations conducted every 3-4 nights in dark time, allowing the construction of high-quality multicolor SN light curves. Supplementary $u^{*}$-band data are also acquired, although these data are not time sequenced. SN candidates are observed spectroscopically at the ESO VLT, Gemini, or W. M. Keck telescopes to confirm their nature and to measure a spectroscopic redshift either from host galaxy features or from the SN itself (see Howell et al. [2005] for an overview of the spectroscopic typing). A description of the real-time search operations and the criteria for following $\mathrm{SN}$ candidates spectroscopically can be found in Sullivan et al. (2006).

The survey is now in its third year, and each of the four survey fields has now been observed for at least two complete "seasons," each spanning the 5-6 months the field is visible from Mauna Kea. This paper uses data from the first two seasons of D1, D2, and D4, as well as the second and third seasons of D3. (The first season of D3 was performed in a presurvey period where the data quality and spectroscopic completeness were substantially lower.) During this period, 116 spectroscopically confirmed $\mathrm{SNe}$ Ia were obtained over the redshift range $0.2<z<0.75$, of which 100 were detected during the period of the survey for which an accurate rate efficiency calculation can be performed (see Neill et al. [2006] and our $\S$ 4.2). To this confirmed SN Ia sample, we add 24 probable $\mathrm{SNe}$ Ia (see $\S 4.2$ ) that lack a spectroscopic identification but for which a reliable SN Ia photometric redshift can be estimated and that possess an excellent light-curve fit to a SN Ia. This set of $124 \mathrm{SNe}$ Ia forms the primary sample studied here.

\subsection{Galaxy Data}

The images that we use to measure the galaxy parameters are also constructed from data taken as part of the CFHTLS. Deep optical stacks were generated for each filter $\left(u^{*} g^{\prime} r^{\prime} i^{\prime} z^{\prime}\right)$ in each field from "Elixir" processed images (Magnier \& Cuillandre 2004) available from the Canadian Astronomy Data Centre 
(CADC).$^{13}$ We summarize the main details of our processing steps here. A precise astrometric solution, accounting for distortion, is assigned to every image frame, and the seeing and photometric quality is determined by flux measurements of tertiary standard stars in the CFHTLS fields compiled by the SNLS team. Any two-dimensional sky variation is removed from each frame by fitting the background spatially and subtracting the resulting fit. Each individual frame has a weight map associated with it, containing the uncertainty in each pixel from considerations of photon noise from the sky background and object photons. Known bad pixels (as determined from the Elixir flat fields) and saturated pixels are assigned a weight of zero.

For the SN host galaxies, we require deep optical stacks with no SN light present when we measure the fluxes of a given object (the presence of SN light would otherwise bias the galaxy flux measurements). We therefore generate stacks on a "per season" basis; stacks for season one $\mathrm{SNe}$ are generated using only data taken in season two and later seasons, and so on. The period between observing seasons for each field -6 to 7 months - allows ample time for SN light to have faded to an negligible level. Every image is resampled to a common pixel coordinate system, excluding images that do not meet the data quality criteria for a given field/filter/season combination. The goal is to maximize exposure depth while retaining excellent seeing in the final stacks (hence, different seeing cuts were used for each field/filter/season to account for varying observing conditions at different times of each year). We also require that, for a given field/season combination, the stacks in each filter have a similar seeing. The typical seeing of the final stacks is $0.7-0{ }^{\prime \prime} 8$. The SWARP package (ver. 2.15.6) with a LANCZOS3 kernel is used for the resampling. The resulting resampled frames are combined using a weighted average, with a sigma clipping to remove artifacts (from satellite trails and cosmic rays) using a custom-written routine.

We also require flux information for the general galaxy population to act as a comparison sample to the SN Ia host galaxies in later sections. For this we use data from the two fields for which the deepest data exist (D1 and D4), particularly in $u^{*}$. We use the same stacking algorithm as above, but produce stacks including all the data for a given field - the field galaxies have no contaminating SN light to concern us-hence, these stacks are deeper than those used for the host galaxies.

Photometric zero points are derived from observations of standard stars taken from Smith et al. (2002) and transformed to the Sloan Digital Sky Survey (SDSS) photometric system; these zero points are determined separately for each of the deep fields in the month in which the photometric reference epoch is defined. Two of the fields (D2 and D3) are in the SDSS, and this provides an independent check of the zero points. The CFHTLS observes in a filter system close to the Smith et al. (2002) $u^{\prime} g^{\prime} r^{\prime} i^{\prime} z^{\prime}$ SDSS system, although the MegaCam $u^{*}$ filter differs from the SDSS $u^{\prime}$ filter, being designed to take advantage of the improved UV capabilities of CFHT and MegaCam. Our effective filter responses can be found in Sullivan et al. (2006).

\subsection{Host Galaxy Identification and Flux Measurement}

The correct host galaxy for every SN Ia in our sample is identified as follows. We begin by measuring the $\mathrm{SN}$ position from point-spread function (PSF) fits to the SN in subtraction images on epochs when the signal-to-noise ratio is largest. These SN positions are accurate to a fraction of a pixel, or $\simeq 0$ ". 02 (one MegaCam pixel is 0"186). By converting these pixel positions to

13 See http://cadcwww.dao.nrc.ca/.

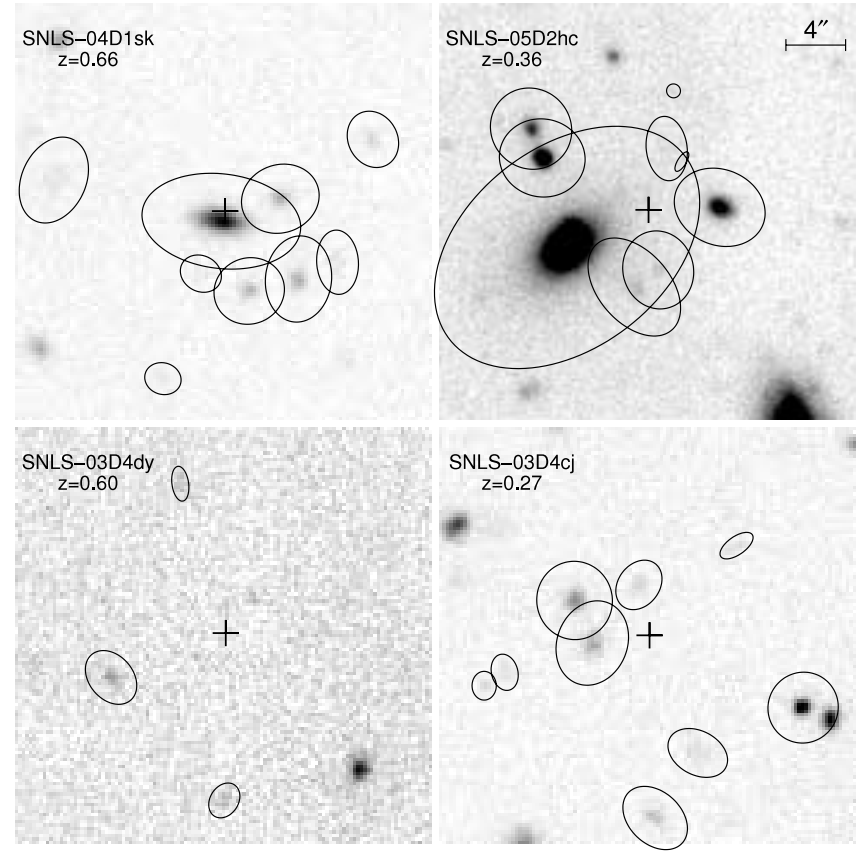

FIG. 1.-Examples of the SN Ia host galaxy identification technique used in this paper (see $\S 2.3$ ). Four SNe are shown. In each panel, the $\mathrm{SN}$ position is marked with a cross, and each candidate host, as detected by SExtractor, has the $5 R$ ellipse overplotted (see $\S 2.3$ for the definition of $R$ ). The nearest host in terms of this $R$-parameter is considered to be the correct host; $\mathrm{SNe}$ with no hosts inside $5 R$ are considered "hostless." Top left: SNLS-04D1sk, a straightforward case in which the identification is unambiguous. Top right: SNLS-05D2hc, where the nearest host in terms of arcseconds is probably not the correct identification. Bottom left: SNLS-03D4dy, where no potential host is found within several arcseconds of the SN position. Bottom right: SNLS-05D2hc, where all candidate hosts lie at $R>5$.

right ascension and declination, we can then precisely identify the location of the SN on the $u^{*} g^{\prime} r^{\prime} i^{\prime} z^{\prime}$ deep stacks even though no SN light is present, as all the frames share a common astrometric system. For each SN Ia, we use SExtractor, version 2.4.4 (Bertin \& Arnouts 1996; see Holwerda [2005] for an excellent user guide) to identify all potential host galaxies within $10^{\prime \prime}$ of the SN position and simultaneously measure photometric information on the host galaxies. SExtractor is used in dual-image mode for the photometric measurements; detections are performed in the $i^{\prime}$ filter (the filter with the deepest data) and measurements performed in each of $u^{*} g^{\prime} r^{\prime} i^{\prime} z^{\prime}$.

Various photometric measurements and galaxy structural parameters are recorded for each galaxy. We use the SExtractor MAG_AUTO flux measures and associated errors throughout this paper. The MAG_AUTO aperture is a flexible elliptical aperture (e.g., Kron 1980) with a characteristic "Kron" radius; we configure SExtractor to measure fluxes inside 2.5 Kron radii. Analytically, this results in $\sim 90 \%$ of an object's light being measured in each aperture (Infante 1987; Graham \& Driver 2005), although this may break down for very faint, highly concentrated galaxies at the detection limit (Bernstein et al. 2002; Benítez et al. 2004; see discussion in Graham \& Driver [2005]). The use of SExtractor in dual-image mode (and the similar PSFs of the stacks in the different filters) ensures that the same size MAG AUTO aperture is used for the different measurements of a given galaxy, minimizing aperture mismatches. Statistical errors in each flux measurement are estimated using the weight image of the final stack as an rms image in SExtractor.

The identification of the correct host galaxy is not always straightforward - occasionally the closest host in arcseconds is 
probably not the correct identification. Instead we use a separation normalized by the apparent size of the galaxy to which the $\mathrm{SN}$ is being compared (Fig. 1). We calculate the separation of the SN from each candidate host galaxy in terms of the elliptical radius $(R)$ along a line connecting the SN pixel position $\left(x_{\mathrm{SN}}, y_{\mathrm{SN}}\right)$ to a given host center $\left(x_{\mathrm{gal}}, y_{\mathrm{gal}}\right)$. The elliptical shape is determined by SExtractor, defined by semimajor $\left(r_{A}\right)$ and semiminor $\left(r_{B}\right)$ axes together with a position angle $(\theta)$, with $R$ given by

$$
R^{2}=C_{x x} x_{r}^{2}+C_{y y} y_{r}^{2}+C_{x y} x_{r} y_{r},
$$

where $x_{r}=x_{\mathrm{SN}}-x_{\mathrm{gal}}, y_{r}=y_{\mathrm{SN}}-y_{\mathrm{gal}}, C_{x x}=\cos ^{2}(\theta) / r_{A}^{2}+\sin ^{2}(\theta) / r_{B}^{2}$, $C_{y y}=\sin ^{2}(\theta) / r_{A}^{2}+\cos ^{2}(\theta) / r_{B}^{2}$, and $C_{x y}=2 \cos (\theta) \sin (\theta)\left(1 / r_{A}^{2}+\right.$ $\left.1 / r_{B}^{2}\right)$. The value $R$ is determined for every candidate host galaxy, the hosts ordered by $R$, and each $\mathrm{SN}$ is assigned to the host that is nearest in terms of this parameter. The isophotal limit of a given object corresponds to $R \sim 3$; we consider only galaxies with $R \leq 5$. In some cases ( $\sim 7 \%)$, no host galaxy is identified by SExtractor with $R \leq 5$; we then measure the flux and flux error inside a 3 " diameter aperture centered on the SN position and use this as the "galaxy" flux in what follows. Figure 1 shows a visualization of the technique for four SNe Ia.

We also measure the properties of the general galaxy population using the deeper stacks constructed as described above. We use SExtractor with the same parameters as for the SN host galaxies.

\section{GALAXY PROPERTIES}

This section details the techniques we use for converting the galaxy properties into characteristics that can be used for our subsequent analysis in $\S \S 5$ and 6 . We first discuss the technique of converting the observed fluxes of $\S 2.3$ into galaxy stellar masses and SFRs, both for the host galaxies themselves and for the general field galaxy population. We then detail a number of consistency checks we perform on these derived parameters.

\subsection{Galaxy SED Fitting}

In order to investigate $\mathrm{SNe}$ Ia in relation to the mean properties of the stellar population from which they were formed, we need to derive properties such as stellar mass and SFR for the galaxies. To do this we fit a series of template galaxy SEDs to the broadband fluxes available for each galaxy $(\S 2.3)$ and then use the best-fit SED for the estimation of the various properties (see, for example, Brinchmann \& Ellis 2000). This technique is similar to that employed by photometric redshift codes (e.g., Gwyn \& Hartwick 1996; Bolzonella et al. 2000; Le Borgne \& RoccaVolmerange 2002). The best-fit SED is determined using a $\chi^{2}$ minimization between the observed fluxes, the corresponding flux errors, and the synthetic photometry generated by integrating the template SEDs through the SNLS effective filter responses. For the host galaxies, the spectroscopic redshift is known from the SN confirmation spectrum and held fixed, reducing the uncertainty in the derived properties of each galaxy. For the field population no spectroscopic information is available, and the redshift is left as a free parameter in the fits.

Our set of synthetic templates is computed with the PÉGASE. 2 galaxy spectral evolution code (Fioc \& Rocca-Volmerange 1997; Le Borgne \& Rocca-Volmerange 2002; Le Borgne et al. 2004). This code and the SEDs that it generates have been used extensively in the literature to constrain the properties of high-redshift galaxies (e.g., Glazebrook et al. 2004; McCarthy et al. 2004; Grazian et al. 2006). We use eight scenarios that evolve self-consistently with age; details of the physical parameters defining them can be found in Table 1 of Le Borgne \& Rocca-Volmerange (2002). We assume a Kroupa (2001) initial mass function (IMF) and assume the IMF is universal across environment (see discussion in Kroupa [2002]). The synthetic SEDs are the sum of light emitted by stars and nebular emission (continuum and lines), including attenuation by dust with a King model or a plane-parallel slab geometry. The quantity of dust evolves consistently with the amount of gas present in the galaxy.

A SED is computed at 69 time steps in each of the eight scenarios, giving a total of 552 template SEDs. When fitting a given galaxy, we use only templates younger than the age of the universe at the redshift of the galaxy.

\subsection{Galaxy Derived Properties}

Two physical parameters, for both the SN Ia host galaxies and for the general galaxy population, are of particular interest in this study: the galaxy mass and the galaxy SFR. The first is the total stellar mass of a galaxy, the total current mass in stars of all types and ages. This is derived by integrating the total star formation history (SFH) of the best-fit scenario up to the best-fit age and subtracting the mass of stars that have died.

The second parameter is the amount of recent star formation that the galaxy has experienced. There are several approaches that could be taken. First, one could simply divide the ultraviolet (UV) luminosity of each galaxy by a conversion factor to obtain a UVderived SFR (e.g., Donas et al. 1987). However, such an approach tends to overestimate the SFR in old stellar systems due to the contaminating presence of evolved stars. A second approach to determining instantaneous SFRs is to use nebular emission lines (Kennicutt 1998). Unfortunately, only very limited information on the appropriate lines is available for our host sample from the SN confirmation spectra, with large uncertainties from aperture losses, SN light contamination, and flux calibration.

We instead estimate the mean SFR from our best-fitting scenarios, averaging the SFR over a longer time interval. This provides an automatic correction for the UV light from old evolved stars. To define the period over which the star formation should be averaged, we carried out simulations in which the mean SFR was estimated from synthetic $u^{*} g^{\prime} r^{\prime} i^{\prime} z^{\prime}$ photometry measured both on the idealized models and on models with stochastic SFHs (see $\S 3.3$ ). These simulations showed that mean SFRs on periods of 0.5 Gyr can be reliably recovered without significant systematic errors. On shorter timescales systematics can be introduced, particularly for galaxies for which the redshift is not known (see Table 1 and discussion in $\S 3.3$ ).

For the general galaxy population, an alternative approach would be to use a parameterized form of the mass or luminosity functions, either directly inferred from high-redshift spectroscopic surveys (e.g., Ilbert et al. 2005; Willmer et al. 2006; Bundy et al. 2005) or low-redshift functions adjusted for evolution to high redshift. While this is possible for the global mass function, no equivalent distribution exists for the SFR property of field galaxies, requiring a conversion from (for example) a rest-frame $U$-band or UV luminosity function. Furthermore, since a goal of this work is to investigate host galaxy properties binned by the type of the host galaxy $(\S 5)$, mass and luminosity functions derived from galaxies categorized in the same way would be required, and these are not currently available.

Uncertainties in the derived parameters for our galaxies arise from both statistical and systematic errors (discussed in $\S 3.3$ ). Statistical errors derive from the photometric measurements of the galaxies and are accounted for by considering the full range in the quality of the 552 template fits (as defined by the $\chi^{2}$ statistic). This maps out a probability surface in the stellar mass and SFR parameters determined from the fits, and allows estimates 
TABLE 1

Input and Recovered Masses and SFRs for Our Simulated Galaxies

\begin{tabular}{|c|c|c|c|c|c|c|}
\hline \multirow[b]{2}{*}{ QUANTITY } & \multicolumn{3}{|c|}{ Redshift Known } & \multicolumn{3}{|c|}{ Redshift Free } \\
\hline & Offset $^{\mathrm{a}}$ & $\begin{array}{c}\text { Standard } \\
\text { Deviation }\end{array}$ & $\begin{array}{c}\text { Fraction of } \\
\text { Outliers }\end{array}$ & Offset & $\begin{array}{l}\text { Standard } \\
\text { Deviation }\end{array}$ & $\begin{array}{c}\text { Fraction of } \\
\text { Outliers }\end{array}$ \\
\hline Mass ... & -0.11 & 0.31 & 0.042 & -0.02 & 0.29 & 0.101 \\
\hline$\overline{\mathrm{SFR}}(0.05)^{\mathrm{b}}$ & 0.11 & 0.51 & 0.003 & 0.39 & 0.51 & 0.032 \\
\hline$\overline{\operatorname{SFR}}(0.10)^{\mathrm{b}}$ & 0.04 & 0.47 & 0.003 & 0.32 & 0.55 & 0.026 \\
\hline$\overline{\operatorname{SFR}}(0.20)^{\mathrm{b}}$ & -0.04 & 0.43 & 0.006 & 0.23 & 0.55 & 0.023 \\
\hline$\overline{\mathrm{SFR}}(0.50)^{\mathrm{b}}$ & -0.10 & 0.46 & 0.003 & 0.11 & 0.59 & 0.026 \\
\hline$\overline{\mathrm{SFR}}(1.00)^{\mathrm{b}}$ & -0.14 & 0.52 & 0.031 & 0.02 & 0.58 & 0.076 \\
\hline
\end{tabular}

a All numerical values are in dex units.

${ }^{\mathrm{b}}$ Values in parentheses are in gigayear units.

of the statistical uncertainties of each of the derived physical parameters; lower signal-to-noise ratio photometry will be consistent with a wider range of fit templates and hence a wider range of stellar masses and SFRs. Invariably, the derived masses and SFRs possess nonsymmetrical error bars, particularly for the fainter systems. In some cases, fainter galaxies can have more than one minimum in the $\chi^{2}$ space. In this case, the best fit is considered as the solution, but the error bars in mass and SFR are extended to the largest interval of uncertainty, covering the range of parameters between the minima. The errors are therefore conservative for galaxies with several minima.

\subsection{Systematic Errors}

Systematic errors in our derived galaxy stellar masses and mean SFRs potentially arise from many sources. These include the wavelength range of our input photometry, and our choice of spectral libraries, IMFs and SFHs that form the template SEDs used for the fitting, and are of course harder to estimate. We first test the accuracy of the photometric redshift estimates by running fits to the host galaxies (with known spectroscopic redshifts) with the redshift left as a free parameter. Figure 2 (left) shows these spectroscopic and photometric redshift estimates for the SN Ia host galaxies. For galaxies with SExtractor detections, the median of $|\Delta z|=\left|z_{\text {spec }}-z_{\text {phot }}\right|$ is 0.02 (the 90th percentile is 0.15 ), and the median of $|\Delta z| /\left(1+z_{\text {spec }}\right)$ is 0.012 (90th percentile is 0.09 ). These measures contain photometric redshifts from galaxies with a significant photometric uncertainty due to their faintness (and hence uncertainty in the photometric redshifts). To confirm that these error estimates are reasonable, we calculate the dispersion $|\Delta z| / \sigma$, where $\sigma$ is the appropriate error in the photometric redshift; $90 \%$ of the photometric redshift estimates lie within $2 \sigma$ of their corresponding spectroscopic redshift.

We next compare the host galaxy properties derived from the template fit with a known redshift against those derived from the photometric redshift fit (cf. Fig. 3 of Bundy et al. 2005). Such a comparison will show whether the photometric redshift properties of the galaxies we study can reliably trace the true galaxy properties in the mean. We compare the stellar mass and mean SFR estimates for galaxies using the spectroscopic redshift with the same estimates when the redshift is allowed to float (Fig. 3). The agreement for both quantities is reasonable, with the mass estimates appearing more robust. No mass or SFR-dependent trends are seen. The median difference $\Delta M=M_{\text {spec }}-M_{\text {phot }}$ is 0.026 dex $(90 \%$ of $\Delta M$ lie within $0.2 \mathrm{dex})$, and the difference
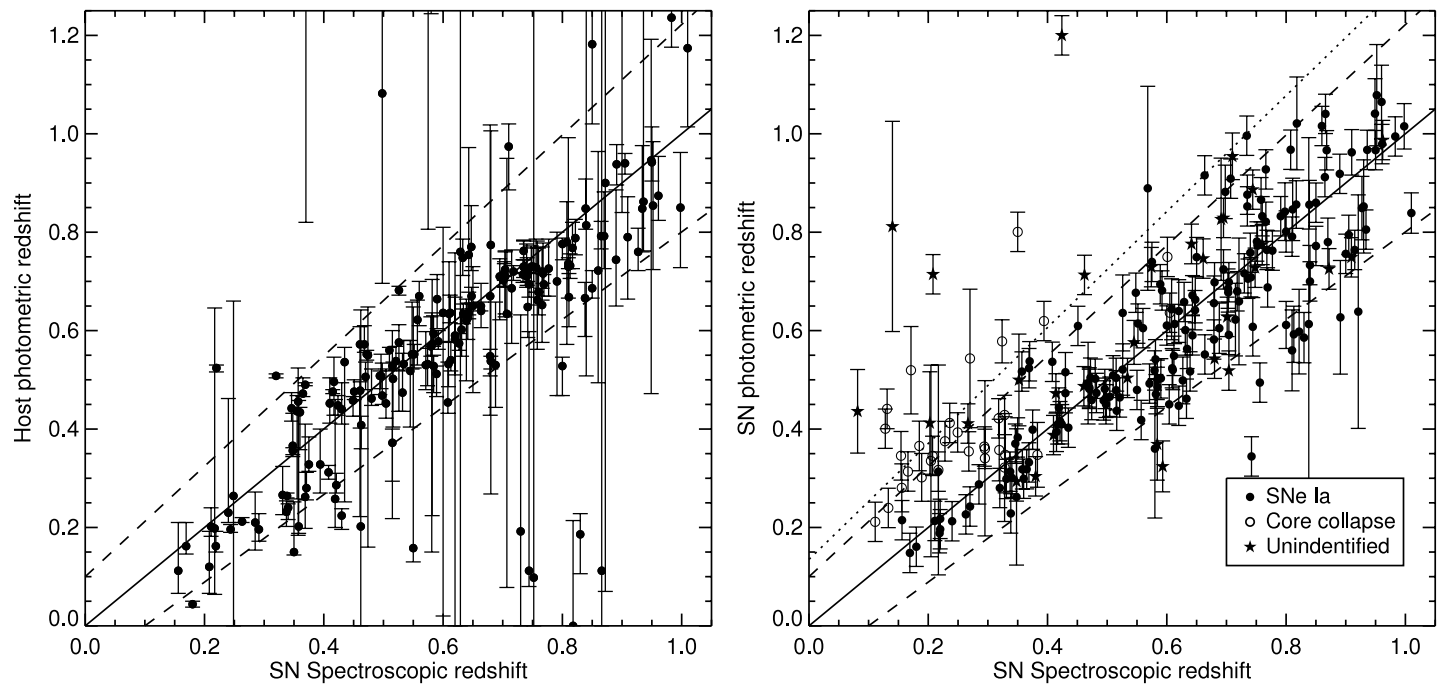

FIG. 2.-Comparison of spectroscopic and host galaxy photometric redshift estimates for the SNLS SN Ia host galaxies (left) and the comparison between spectroscopic and SN photometric redshift estimates for all SNLS confirmed SNe (right). The solid line shows a 1:1 agreement between $z_{\text {spec }}$ and $z_{\text {phot }}$; the dashed lines show a $10 \%$ uncertainty in $\left(1+z_{\text {spec }}\right)$. In the right panel, the dotted line shows the region above which SNe were excluded as SNe Ia during the SN completeness correction stage ( $(4.2)$. The SN Ia photometric redshifts follow the method of Sullivan et al. (2006). For the host galaxy photometric redshift, the median of $z_{\text {spec }}-z_{\text {phot }}$ is 0.02 with a standard deviation of 0.15 . For the $\mathrm{SN}$ photometric redshifts, these values are -0.007 and 0.088 . Note that the SN Ia photometric redshift estimates include a cosmological prior that precludes their use for determining the cosmological parameters. 

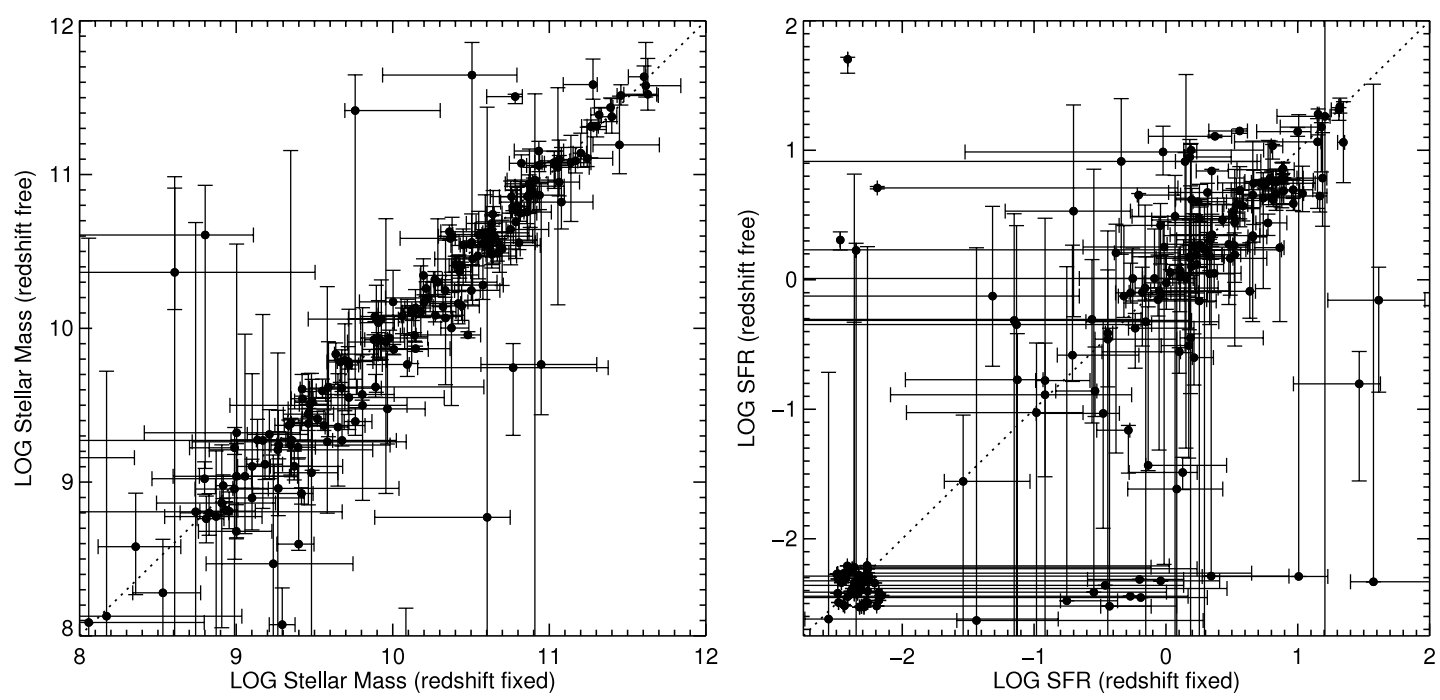

FIG. 3.-Comparison of mass $(M)$ and SFR estimates for the SNLS SN Ia host galaxies when the redshift is fixed (spectroscopic redshift; $y$-axes) and when the redshift is determined using the photometric redshift code, Z-PEG (photometric redshift; $x$-axes). Zero SFR systems are shown with log (SFR) $=-2.5$ plus a random offset for clarity. The average properties of the sample as determined when the redshift is known vs. the photometric redshift estimate are very similar; the median difference $\Delta M$ is 0.026 dex ( $90 \%$ of $\Delta M$ lie within 0.2 dex), and the difference $\Delta$ SFR is $0.034 \operatorname{dex}(90 \%$ of $\Delta$ SFR lie within 0.38 dex).

$\Delta \mathrm{SFR}$ is $0.033 \mathrm{dex}(90 \%$ of $\Delta \mathrm{SFR}$ lie within $0.38 \mathrm{dex})$. A comparison of the mass function derived from our photometric redshift fits to published mass functions of field galaxies will be presented in an upcoming paper (D. Le Borgne et al. 2006, in preparation).

The next potential source of concern is the lack of near-IR data and the impact on our estimates of stellar masses, and the lack of very short wavelength data and the impact on the derived SFRs. These could be particularly pronounced in systems with both a young and an old stellar population, such as those galaxies experiencing recent star formation events. Clearly, the uncertainties in derived stellar masses and mean SFRs will be larger in the absence of UV and near-IR data; however, as long as the error bars in these properties reflect this, this additional uncertainty will be carried through in our analysis. A larger concern would be any systematic under- or overestimation of the stellar masses and SFRs.

We investigate this by using our standard templates to fit photometry generated from SEDs with stochastic SFHs. Synthetic SEDs are formed from the composite of three random exponential SFHs each with a different age $(t)$, mass fraction, and $\tau$. One SFH is constrained to be old $(t>1 \mathrm{Gyr})$, one of intermediate age (200 Myr $<t<2 \mathrm{Gyr}$ ), and one young (30 Myr $<t<$ $100 \mathrm{Myr})$. Each of these three random SFHs is converted into a SED at the randomly selected $t$, and the three SEDs added together to form one composite SED. We place this composite SED at a random redshift $(0.2<z<0.75)$, generate synthetic $u^{*} g^{\prime} r^{\prime} i^{\prime} z^{\prime}$ photometry, and see how well PÉGASE. 2 recovers the stellar mass and mean SFR using the eight idealized scenarios of $\S 3.1$ both when the redshift is fixed and when it is left as a free parameter.

The results are given in Table 1 and are encouraging. For the stellar masses, we see only small differences in the mean between the input and recovered value with a standard deviation of around 0.3 dex. For the mean SFRs, again there is no significant systematic offset, although the standard deviation of the differences is larger particularly when the redshift of the galaxy is not known.

We also briefly investigate the effect of dust on our stellar mass and mean SFR estimates. Although dust is included in the idealized scenarios (see our $\S 3.1$ and discussion in Le Borgne \& Rocca-Volmerange [2002]), this will only provide an average cor- rection to our derived properties. We experimented with adding extra dust to our simulated SEDs [up to $E(B-V)=0.5$, using a Calzetti et al. (2000) extinction law]. As expected, this can lead to a systematic underestimation of the mean SFR (up to 0.4 in dex in some cases) and a small increase in the standard deviation, although the accuracy of the stellar mass estimates was not affected.

Finally, we estimate systematic uncertainties by deriving the galaxy properties using a similar technique but different templates. We use the photometric redshift code of Gwyn \& Hartwick (1996), which uses a different set of (empirical) galaxy templates (Coleman et al. 1980; Kinney et al. 1996) to those used in Z-PEG. We determine masses by fitting Buzzoni (2005) population models to the best-fitting templates, which results in a mass-to-light ratio that can be used to convert the galaxy luminosities into masses. SFRs are determined by dividing the flux at $2800 \AA$ by $4.8 \times$ $10^{27} \mathrm{ergs} \mathrm{s}^{-1} \mathrm{~Hz}^{-1}$ (Buzzoni 2002; Madau et al. 1998b), with a small correction for early-type SEDs to account for the contribution from older stars (see Buzzoni 2002)

The two different techniques for estimating the masses and the SFRs showed a good agreement. The mass and SFR estimates agree to better than a factor of 2 : the mean difference in mass was 0.35 dex (in the sense Z-PEG measured smaller masses) with an rms scatter of 0.24 , and the mean offset for the SFR estimates was 0.15 dex (in the sense Z-PEG measured larger SFRs) with an rms scatter of 0.29 . Most importantly, no mass-dependent or SFR-dependent trends were detected. Given that Z-PEG is used to calculate masses and SFRs for both field and host galaxies, these offsets in mass or SFR will cancel in our analyses.

\section{INCOMPLETENESS CORRECTIONS}

In the next two subsections we discuss incompleteness in our sample. We first discuss the incompleteness of our field galaxy population due to the limiting magnitude of our deep field stacks, an effect common to all galaxy redshift surveys. We then discuss the incompleteness of our SN Ia sample.

\subsection{Galaxy Incompleteness Corrections}

Incompleteness will affect our stellar mass and mean SFR distribution functions of the field galaxy population. Our survey is magnitude limited; galaxies with a given absolute magnitude (and 
spectral type) will become fainter than this magnitude limit at different redshifts, which may be less than the largest redshift we consider here $(z=0.75)$. We adopt the traditional $V_{\max }$ method (e.g., Schmidt 1968; Felten 1976) to correct for this effect. The value $V_{\max }$ is defined as the comoving volume within which each galaxy - as defined by its absolute magnitude and $k$-correction from best-fitting SED template-would remain in our sample, i.e., within which it would satisfy the limits in apparent magnitude of the current depths of the optical stacks. Each galaxy is then weighted by a factor $V_{\text {survey }} / V_{\max }$ when computing the various mass and SFR distributions used in $\S 5$, where $V_{\text {survey }}$ is the total survey volume.

The SExtractor photometric measurements are performed by detecting in the $i^{\prime}$ filter, and the depth in this filter defines the limiting magnitudes. The limiting magnitudes on the $i^{\prime} \mathrm{D} 1$ and D4 stacks are determined by inserting fake sources into the stacks and measuring the fraction recovered by SExtractor as a function of magnitude; the limiting magnitude is that at which $50 \%$ of fake sources are recovered.

\subsection{SN Incompleteness and Rate Calculation}

Incompleteness will affect our SN Ia sample in several ways. First, we could miss SNe Ia altogether due to inefficiencies in our search pipeline (the detection efficiency). Second, detected SNe Ia in the redshift range of interest could be lacking a spectroscopic observation because such an observation would be too challenging, for example, due to host galaxy contamination or the SN maximum light falling during a bright moon period. Finally, SNe Ia could lack a spectroscopic confirmation because poor weather affected the spectroscopic scheduling.

To correct for these effects, we adopt the scheme of Neill et al. (2006), who calculated the SNLS SN Ia rate over $0.2<z<0.6$, but we extend the calculation to $z=0.75$. The first source of incompleteness, detectability, is accounted for using simulated SN data. Fake SNe are placed in real SNLS data, and the recovery efficiency as a function of SN brightness, position, sky background, host brightness, exposure time, seeing, and sky transparency is calculated (see Neill et al. 2006).

We also determine the observing window within which maximum light of a SN Ia would have to occur in order to be considered for a spectroscopic observation and for which we can derive a reliable photometric classification (see below). SN Ia candidates detected at the start or end of an observing season lack a full light curve and therefore are not usually observed spectroscopically, as any resulting light-curve fit would be far more uncertain. For the purposes of this study, these cutoffs are defined as follows. (1) The SN must have at least two $i^{\prime}$ and at least one $r^{\prime}$ observation between -15 and -1.5 days in the SN rest frame, (2) there must be at least $1 g^{\prime}$ observation between -15 and +5 days, and (3) there must be at least $1 r^{\prime}$ or $i^{\prime}$ observation after +11.5 days but before +35 days. These criteria cull $\mathrm{SNe}$ Ia whose light curves do not properly sample maximum light and for which a light-curve width measurement would be correspondingly more uncertain, and for which a color near maximum was not measured. Any confirmed $\mathrm{SNe}$ Ia that do not meet these criteria are excluded from this analysis; this reduces our sample from 116 SNe Ia to 100 . Our simulations then provide, for a given field observing season, the efficiency required to convert an observed per season rate (the number of SNe Ia with $0.2<z<0.75$ meeting the light-curve criteria defined above) into a yearly rate. These efficiencies can be found in Table 2.

The spectroscopic incompleteness - the fraction of candidate $\mathrm{SNe}$ Ia detected but never observed spectroscopically-is the
TABLE 2

Breakdown of Candidate Numbers and Efficiencies

\begin{tabular}{|c|c|c|c|c|c|c|}
\hline \multirow[b]{2}{*}{ FIELD $^{a}$} & \multirow{2}{*}{$\begin{array}{c}\text { AREA } \\
\left(\operatorname{deg}^{2}\right)\end{array}$} & \multirow[b]{2}{*}{$N_{\text {spec }}{ }^{\mathrm{b}}$} & \multirow[b]{2}{*}{$N_{\text {phot }}{ }^{\mathrm{c}}$} & \multicolumn{3}{|c|}{ EFFICIENCY } \\
\hline & & & & $0.2-0.4$ & $0.4-0.6$ & $0.6-0.75$ \\
\hline D1 ................... & 1.024 & 25 & 5 & 0.314 & 0.297 & 0.241 \\
\hline D2 .................. & 1.026 & 27 & 4 & 0.223 & 0.218 & 0.181 \\
\hline D3.................... & 1.029 & 28 & 11 & 0.331 & 0.310 & 0.248 \\
\hline D4................... & 1.027 & 20 & 4 & 0.307 & 0.317 & 0.274 \\
\hline
\end{tabular}

${ }^{\text {a }}$ Each field comprises SNe from 2 yr of observing.

b Spectroscopically confirmed SNe Ia with a confidence index from 3 to 5 (Howell et al. 2005).

c SNe Ia with either a spectroscopic redshift but no definitive type, or solely a photometric redshift (see $\S 4.2$ ).

most challenging source of uncertainty to address in this study. We assess the incompleteness using the photometric SN selection method presented in Sullivan et al. (2006). This technique fits SN light curves in the absence of a spectroscopic redshift and returns the best-fitting SN Ia parameters (redshift, stretch, $E_{B-V}^{\text {host }}$, and a dispersion in the SN peak magnitude, $d m$ ), as well as a guide that the candidate under study is a SN Ia. The software was run on $g^{\prime} r^{\prime} i^{\prime} z^{\prime}$ photometry for all SN candidates (including those observed and typed spectroscopically) discovered during the period covered by the simulations above. A comparison of SN spectroscopic redshift and SN photometric redshift for all SNLS $\mathrm{SNe}$ Ia can be found in Figure 2. (Note that as the SN photometric redshift code makes an assumption about the cosmology when fitting the light curve, the resulting photometric redshift cannot themselves be used for determination of the cosmological parameters.) The mean difference $z_{\text {spec }}-z_{\text {phot }}$ for all SNe Ia is -0.007 ; the standard deviation is 0.088 . For the SNe Ia included in this study - over $0.2<z<0.75$ and that meet the lightcurve criteria - the standard deviation is 0.077 . Occasionally, a spectroscopic observation of a SN candidate yielded a spectroscopic redshift but no definitive type; in these cases, we still allow the redshift to float for comparison with the spectroscopic redshift.

There were $286 \mathrm{SN}$ candidates without a spectroscopic confirmation with a SN Ia photometric redshift in the range of interest; 80 do not meet the light-curve coverage criteria that we apply to the spectroscopically confirmed sample and are excluded. This ensures that all candidates have a reliable stretch and $g^{\prime}$ observations. We also exclude two SN candidates where the spectroscopic redshift was in disagreement with the photometric redshift where $z_{\text {phot }}>z_{\text {spec }}+0.135\left(1+z_{\text {spec }}\right)$ (Fig. 2). In these cases, the SN spectra were ambiguous and no type could be determined; usually this was because a $\mathrm{SN}$ Ib/c spectrum provided a similar quality fit to the observed spectrum as did a SN Ia.

The remainder (204) were then culled based on their lightcurve parameterization and the $\chi^{2}$ of the light-curve fits in the different observed filters. We exclude $70 \mathrm{SN}$ candidates with a fitted stretch $>1.35$ (very effective at removing SNe IIP) and 75 candidates whose $\chi^{2}$ per degree of freedom in the fit was $>10$. None of our spectroscopically confirmed SNe Ia had fit parameters in either of these ranges (see Fig. 4).

These simple cuts leave 67 candidate SNe Ia. A further visual inspection of the remaining candidates revealed that although many of these had acceptable overall $\chi^{2}$ fits, they were too blue in $g^{\prime}-r^{\prime}$ before +5 days when compared to a SN Ia template. Hence, our final statistical cut removes 35 objects with 

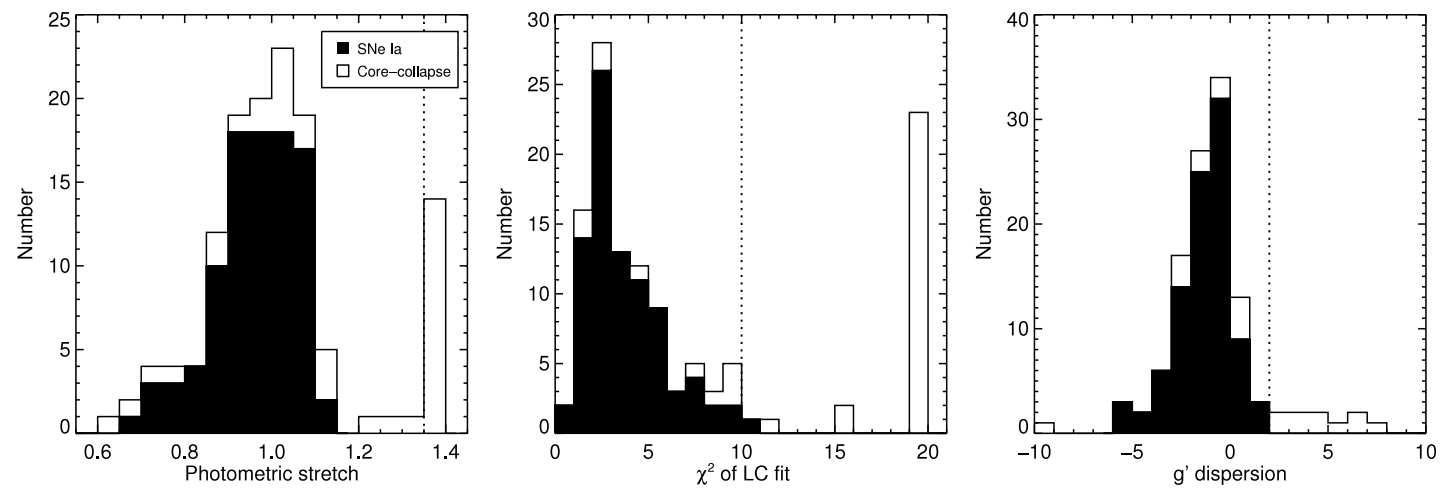

FIG. 4.- Range of fitted stretch (left), light curve $\chi^{2}$ per degree of freedom (center), and early-time $g^{\prime}$ dispersion (right) obtained when running the photometric redshift code of Sullivan et al. (2006) on all spectroscopically confirmed SNLS SNe Ia (filled histograms) and SNLS core-collapse SNe (open histograms) over $0.2<z<0.75$. Cuts of $s>1.35, \chi^{2}>10$, and $g^{\prime}$ mean dispersion $>2$ (see eq. [2]) are effective at removing core-collapse SNe while retaining SNe Ia; these cuts are shown as vertical dotted lines.

a poor $g^{\prime}$ fit at early times $(-15<d<+5)$ or with a $g^{\prime}$ mean dispersion,

$$
\frac{1}{N_{g}} \sum_{i=1}^{N_{g}} \frac{\left(g_{\mathrm{obs}}^{i}-g_{\text {model }}^{i}\right)}{g_{\mathrm{err}}^{i}}>2,
$$

where $N_{g}$ is the number of $g^{\prime}$ light-curve fluxes $g_{\text {obs }}$ with error $g_{\text {err }}$ over $-15<d<+5$, and $g_{\text {model }}$ is the SN Ia template model. This cut does not remove $\mathrm{SNe}$ that are too red (negative dispersions), which could be indicative of extinction, only those that are too blue. The various culls that we use are summarized in Table 3 . This leaves 24 SN Ia candidates to add to the 100 spectroscopically confirmed SN Ia sample; details can be found in Table 2 . For these $\mathrm{SNe}$, the redshift of the $\mathrm{SN}$ Ia is taken to be the SN Ia photometric redshift where no spectroscopic redshift was available. The impact on our results of these photometrically classified SNe Ia is discussed in $\S 5$.

\section{THE SUPERNOVA Ia RATE AS A FUNCTION OF HOST GALAXY PROPERTIES}

In this section, we examine the SN Ia rate as a function of the properties of their host galaxies and use these rates to place constraints on the composition of the total SN Ia rate. We first classify our SNe Ia into three subgroups based on the nature of star formation in their host galaxy. The first group comprises SNe Ia located in host galaxies with a zero mean SFR from the SED fits ("passive" galaxies). We then use the specific SFR, sSFR, defined as the star formation rate per unit stellar mass (e.g., Guzman et al. 1997; Brinchmann \& Ellis 2000; Brinchmann et al. 2004), to classify the star-forming SN Ia host galaxies. With units of per year, sSFR is essentially a measure of the inverse of the formation timescale for a given galaxy: high sSFR galaxies will form

TABLE 3

Breakdown of Photometric Typing Culls

\begin{tabular}{|c|c|c|c|c|}
\hline Criterium & \multicolumn{2}{|c|}{$N_{\text {spec }}$} & \multicolumn{2}{|c|}{$N_{\text {phot }}$} \\
\hline Number over $0.2<z<0.75 \ldots$ & 116 & & 286 & \\
\hline Number rejected on light-curve coverage ........... & $\ldots$ & 16 & $\ldots$ & 80 \\
\hline 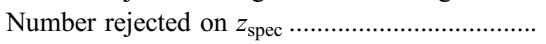 & $\ldots$ & $\ldots$ & .. & 2 \\
\hline Number $\mathrm{r}$ & $\ldots$ & $\ldots$ & $\ldots$ & 70 \\
\hline 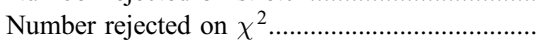 & $\ldots$ & $\ldots$ & $\cdots$ & 75 \\
\hline 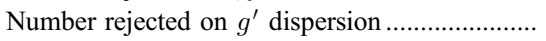 & .. & $\cdots$ & $\ldots$ & 35 \\
\hline Number remaining & 100 & $\ldots$ & 24 & $\cdots$ \\
\hline
\end{tabular}

the mass in their stellar populations on shorter times than low sSFR galaxies. The second group of hosts, defined as $-12.0 \leq$ $\log (\mathrm{sSFR}) \leq-9.5$, have a small or moderate amount of star formation relative to their stellar mass and therefore are likely to possess a substantial evolved stellar component as well as young stars. The third and final group, with $\log$ (sSFR) $>-9.5$, have a large amount of star formation relative to their stellar mass, and therefore stellar populations that have a large component made up of young stars. Broadly speaking, the second group tends to comprise normal star-forming galaxies such as the Milky Way, whereas the third group tends to include vigorously star forming and lower mass dwarf galaxies. The division is illustrated in Figure 5, which shows the distribution of all the SN Ia host galaxies in the stellar mass/SFR plane.

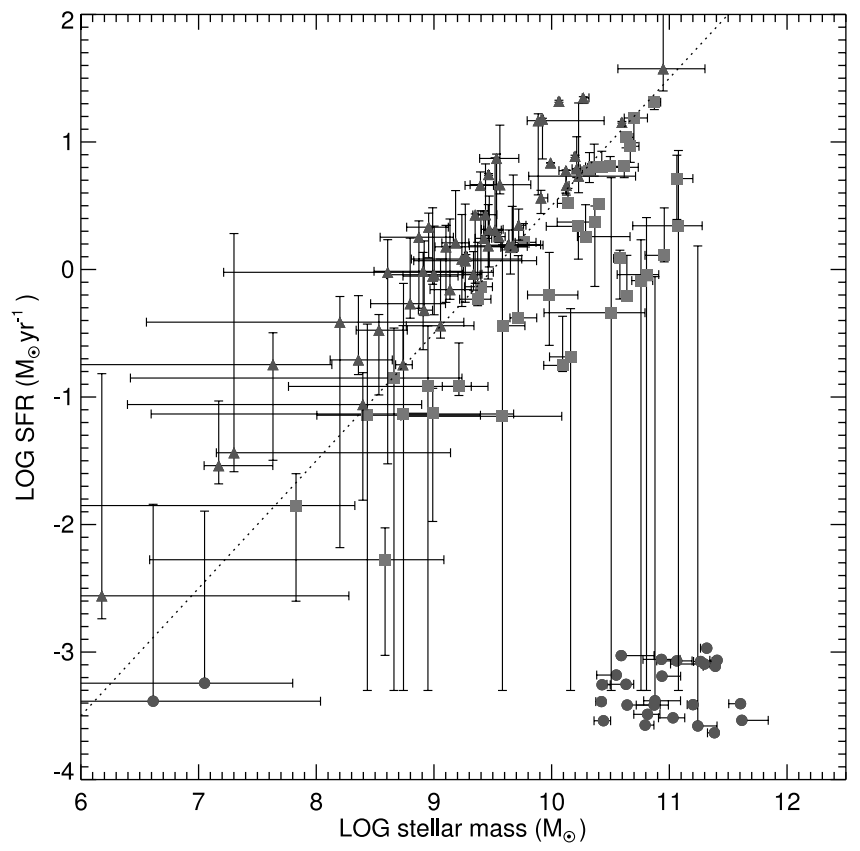

FIG. 5.-Distribution of the SN Ia host galaxies in the SFR-mass plane. Each galaxy is coded according to its assigned type. Passive galaxies are shown as circles, normal star-forming galaxies as squares, and vigorous star formers as triangles. The diagonal dotted line shows the division in specific SFR used to subdivide those hosts that are star forming. The passive galaxies (which have a zero SFR in our models) are assigned a random SFR centered on $0.005 M_{\odot} \mathrm{yr}^{-1}$ for illustration purposes. [See the electronic edition of the Journal for a color version of this figure.] 
An attraction of the SED-fitting technique is that galaxies can be classified according to their star formation properties without regard to morphology. This has some advantages; in morphologically selected samples of distant galaxies, a significant fraction of spheroidals have been shown to possess both "blue cores" and weak O II emission lines in their spectra (Menanteau et al. 2001; Treu et al. 2005), interpreted as a signature of young stars in these galaxies. There is also some evidence for recent star formation in some early-type galaxies from the near-UV color-magnitude relation (Yi et al. 2005). The advantage of the effective color selection of SED fitting is that rather than assume all early-type galaxies consist purely of old stars, the evolutionary model fitting places no a priori constraint on the type of SFH that can be fit to a given galaxy. Young populations are perfectly possible in morphologically spheroidal galaxies, and hence any young stellar population that has a significant impact on a galaxy's colors will be reflected in the best-fitting galaxy SFH.

We examine how the SN Ia rate varies as a function of this specific SFR in Figure 6. The field galaxies are binned according to their value of specific SFR, and the total field galaxy stellar mass in each bin of specific SFR is calculated. We use the $V_{\max }$ technique of $\S 4.1$ to incompleteness correct this distribution. We then bin the SN Ia host galaxies by specific SFR in the same way and calculate the number of SNe Ia per unit stellar mass as a function of the specific SFR of their host galaxies. In Figure 6 the rate in passive galaxies, where the SFR is zero, is shown as a hashed area starting at $\log (\mathrm{sSFR})=-12$ (the height of this hashed area represents the statistical uncertainty in the measurement).

An increase in the rate of SNe Ia per unit stellar mass with increasing specific SFR of the host galaxy is clear. The difference between the rate in passive galaxies and the most vigorous starforming systems is about a factor of 10 ; furthermore, the increase in the rate is a fairly smooth function of the specific SFR. The general trend of Figure 6 can be compared to that observed by Mannucci et al. (2005) in the local universe using a morphologically classified sample of local SNe Ia host galaxies. We illustrate this in Figure 6 by overplotting the Mannucci et al. data on the SNLS results; the Mannucci et al. evolution is shown normalized to the SNLS rate in passive galaxies. The trend is very similar, although there is the obvious caveat that the link between specific SFR and galaxy morphology is not a straightforward one-to-one mapping.

As noted by other authors, this relationship is difficult to reconcile with a model for SNe Ia that originates solely from an old evolved stellar population. Mannucci et al. (2006) and SB05 instead model the $\mathrm{SN}$ Ia rate $\left(\mathrm{SNR}_{\mathrm{Ia}}\right)$ as a composition of two separate components: a prompt component, with a short delay time, and an old component, with a long delay time. The most general form for the $\mathrm{SNR}_{\mathrm{Ia}}$ as a function of time is simply the convolution of the SFR, $\dot{M}_{\text {new }}$, and the probability function for getting a $\mathrm{SN}$ Ia from a stellar population of age $t, P$, i.e.,

$$
\mathrm{SNR}_{\mathrm{Ia}}(t)=\int_{0}^{t} \dot{M}_{\text {new }}\left(t^{\prime}\right) P\left(t-t^{\prime}\right) d t^{\prime}
$$

This rate can be simply modeled by making the assumption that $P$ can be well represented by two components. One has a peak of $B$ at time $t=0$ and is zero at all other times (this represents a very short delay time); the other has $P=A$ constant with time (and represents long delay times), i.e.,

$$
\operatorname{SNR}_{\mathrm{Ia}}(t)=A \int_{0}^{t} \dot{M}_{\text {new }}(t) d t+B \dot{M}_{\text {new }}(t)
$$

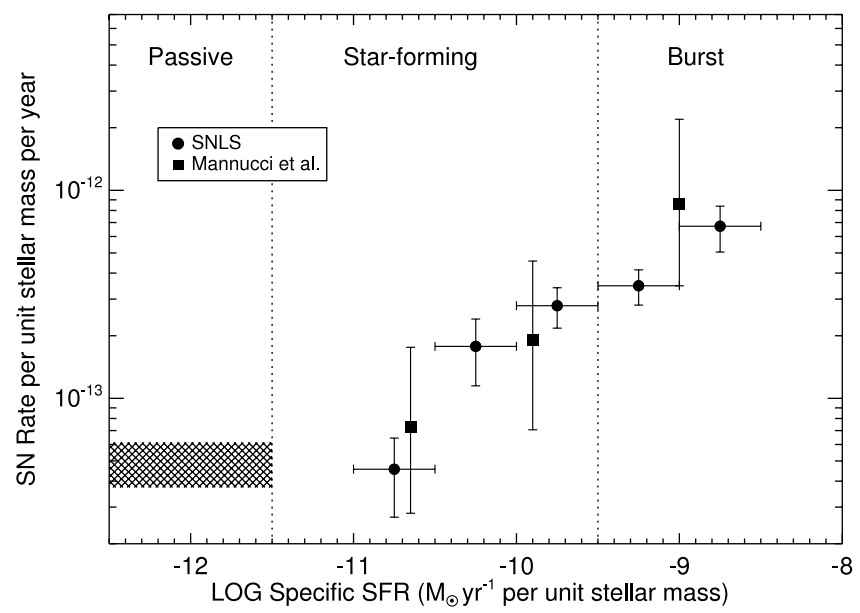

FIG. 6.- Number of SNe Ia per unit stellar mass as a function of the SFR per unit stellar mass of the host galaxy. Points represent SNLS data points in starforming galaxies. The hashed area shows the number per unit stellar mass as measured in the SNLS passive galaxies (assigned zero SFR in our models). Shown for comparison is the evolution in SN Ia rate to later type galaxies observed locally by Mannucci et al. (2005), normalized to the SNLS rate in passive galaxies. The horizontal positioning of the Mannucci et al. data points are subject to a uncertainty when converting their galaxy types into specific SFRs. The vertical dotted lines show the division we use to classify the host galaxies into different types. [See the electronic edition of the Journal for a color version of this figure.]

As $M_{\text {tot }}(t)=\int_{0}^{t} \dot{M}_{\text {new }}(t) d t$, where $M_{\text {tot }}(t)$ is the total mass of a galaxy at time $t$, this equation models the probability of a SN Ia exploding in a given galaxy as depending on both the mass and the instantaneous SFR of that galaxy. The values $A$ and $B$ are constants that relate the total mass and the SFR of a galaxy to the $\mathrm{SNR}_{\mathrm{Ia}}$ in that galaxy (which SB05 fix using the observations of Mannucci et al. [2005]). In effect, $A$ is the $\mathrm{SNR}_{\text {Ia }}$ per unit mass of the old component, and $B$ is the $\mathrm{SNR}_{\text {Ia }}$ per unit SFR of the young component. The model predicts that $\mathrm{SNR}_{\mathrm{Ia}}$ is linear in both host galaxy mass and SFR.

Note that the definition of mass above is slightly different from that measured for the SN host galaxies ( $\$ 3.1$ ). For the hosts, we measure the total stellar mass, i.e., the total mass currently in stars in each galaxy. The value $M_{\text {tot }}$ above is the integral of the SFH for each galaxy - no correction is made for stars that have lost mass at the end of their stellar evolution. The numerical differences in these mass definitions are shown in Figure 7, with the differences being largest in older, lower SFR systems.

The model of equation (4) is a simplification of the real physics. For the prompt component, the model implies a zero delay time between star formation and SN Ia explosion; some nonzero delay time to account for main-sequence lifetime and subsequent accretion onto the white dwarf is obviously required. For the old component, the equation simplifies the complex relationship between the SN Ia delay time and the age of the stellar population by using a simple constant probability rather than a more complex exponential or Gaussian delay time distribution. In reality, for a coeval population, after a few billion years the probability of a SN Ia will likely decrease as the stellar population ages and fewer progenitor stars are available; this could cause an overestimation of the SN Ia rates in the oldest, passive stellar systems.

Yet, these approximations may not be that poor. Mannucci et al. (2006) found that the local SN Ia delay time distribution is well represented by a prompt component modeled as a Gaussian centered at $t=50$ Myr plus a component modeled as an exponential with an $e$-folding time of 3 Gyr. These two terms can be approximated by a delta function at time $t=0$ plus a constant 


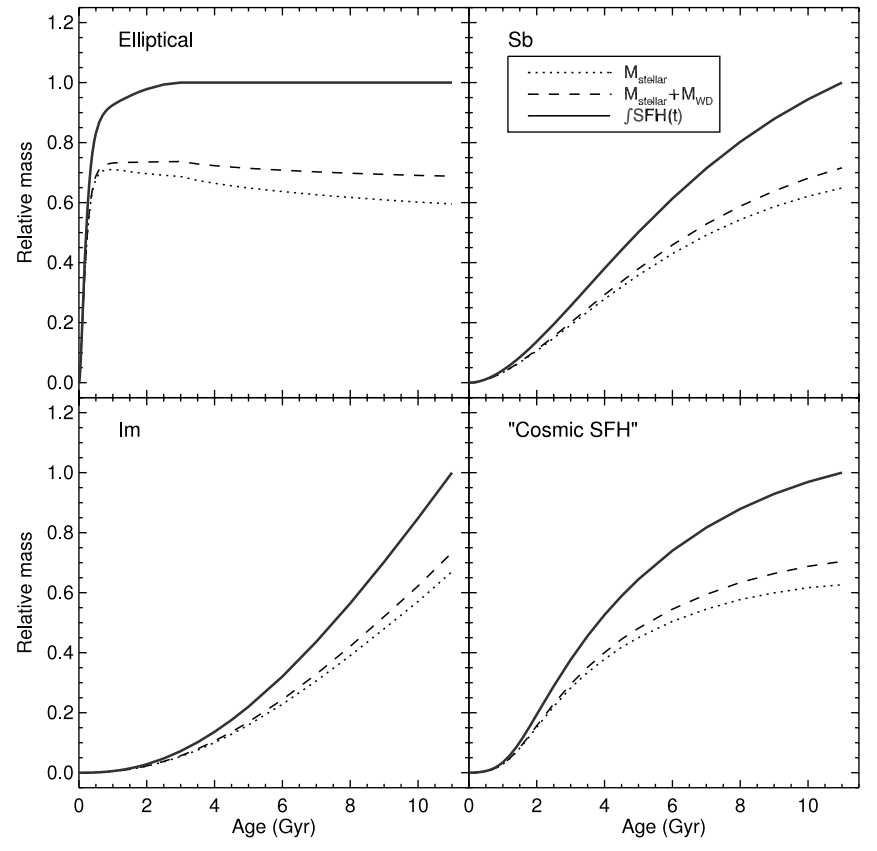

FIG. 7.- Mass evolution, as calculated by PÉGASE.2, for three different galaxy scenarios plus the cosmic SFH of Hopkins \& Beacom (2006). The different lines show the total mass in stars excluding compact objects (dotted line), the total mass in stars plus the mass in white dwarfs (dashed line), and the mass as calculated by simply integrating the SFH of that scenario (solid line). [See the electronic edition of the Journal for a color version of this figure.]

probability thereafter. Furthermore, this equation parameterizes the rate in a convenient form relating to galaxy properties that are relatively straightforward to measure using the SNLS data set introduced above.

In the next sections, we illustrate this model by considering the SN Ia rate as a function of both host galaxy stellar mass and host galaxy SFR taken separately, and then constrain the $A$ and $B$ parameters using a bivariate fit to the mass/SFR data.

\subsection{SN Ia Rate as a Function of Host Galaxy Stellar Mass}

We first attempt to separate any component of the SN Ia rate that may depend on the stellar mass of a galaxy, from any component that depends on the amount of recent star formation, i.e., to separate the two components of equation (4). We can do this using our sample of SNe Ia that exploded in passive galaxies and that in our models have zero recent star formation activity. By binning the SN Ia host galaxies according to their mass, and dividing by the equivalent (incompleteness corrected) distribution of the general galaxy population in each mass bin, we can examine how the probability of a SN Ia explosion is related to the mass of the host galaxy.

We perform this comparison in Figure 8, where we show the dependence of the SN Ia rate as a function of mass of the host galaxy. To account for the (invariably nonsymmetrical) errors in the mass determinations of the SN host galaxies, we have performed a Monte Carlo simulation with 5000 realizations of each SN host with the masses for each host drawn from the estimated probability distribution for that host. We then bin this Monte Carlo population, normalize to the total number of hosts in the sample, and use this binned distribution in our analysis. Such a procedure produces a distribution that accounts for observational statistical uncertainties, which can be considerable in the fainter host subsample where some of the measurements effectively provide only limits on the host parameters.

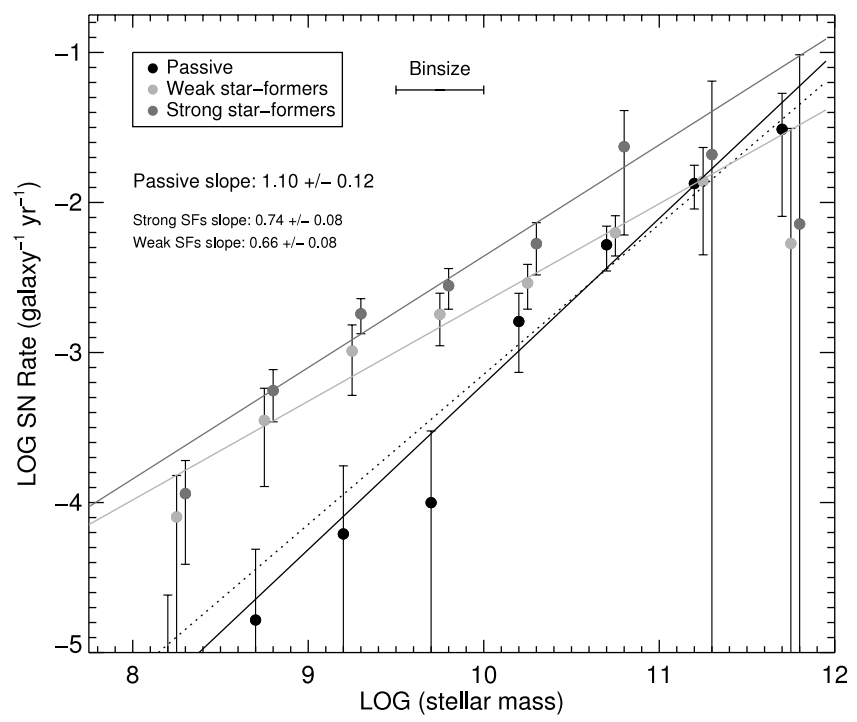

FIG. 8.-Number of SNe Ia per host galaxy as a function of host galaxy stellar mass. The three shades denote SNe Ia in the three different types of host galaxy as partitioned by their specific SFR. The best-fitting lines and slopes to each distribution are shown. For the passive hosts, a line of slope unity is also shown (dotted line). [See the electronic edition of the Journal for a color version of this figure.]

One feature of Figure 8 is the increase in the SN Ia rate with increasing host galaxy mass, present among host galaxies of all types. As the SFR of the passive galaxies is zero (this defines their selection), the contribution from the $B$ term in equation (4) is also zero in these galaxies. Hence, the best-fitting line in loglog space should have a slope of 1 , if equation (4) is a good approximation - the model is linear in mass. For passive galaxies, we find that the slope, $n_{\text {mass }}$, is $n_{\text {mass }}=1.10 \pm 0.12$ with a reduced $\chi^{2}$ of $\simeq 1.14$ (if only spectroscopically confirmed SNe Ia are used in the fits, $n_{\text {mass }}=1.04 \pm 0.13$ ). This implies that the relationship between the SN Ia rate and galaxy mass is consistent with being linear, and the model provides an adequate fit to the data. Using this

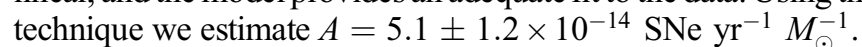

The relationship in later type, star-forming galaxies is different. The best-fitting slopes are statistically consistent: $0.66 \pm 0.08$ (reduced $\chi^{2}=0.77$ ) and $0.74 \pm 0.08$ (reduced $\chi^{2}=0.94$ ) for the low and high specific SFR galaxies, respectively. This translates to an excess of SNe Ia in low-mass star-forming galaxies compared to the passive galaxies, but a similar number in each at the most massive end. This is as expected if the model of equation (4) is correct. Doubling the mass in passive systems will double the SN Ia rate, but doubling the mass in star-forming systems will only double the rate if the SFR doubles as well. As the most massive star-forming systems generally have lower specific SFRs than the lowest mass systems, the increase in the SN Ia rate with host mass in star-forming systems is not linear. The result is that while the prompt component SNe dominate in low-mass starforming galaxies, the old component is more important in higher mass star-forming galaxies.

\subsection{SN Ia Rate as a Function of Host Galaxy Mean SFR}

Having examined the SN Ia rate in galaxies dominated by old stellar populations, we now examine the SN Ia rate in very young populations via the SFR, which we determine as the mean SFR over the last 0.5 Gyr of a galaxy's SFH as determined from the best-fitting template scenario ( $\S 3.1$ ). We first bin both the SN Ia hosts and the general galaxy population by their mean SFR. We incompleteness correct the general galaxy population, and in each 


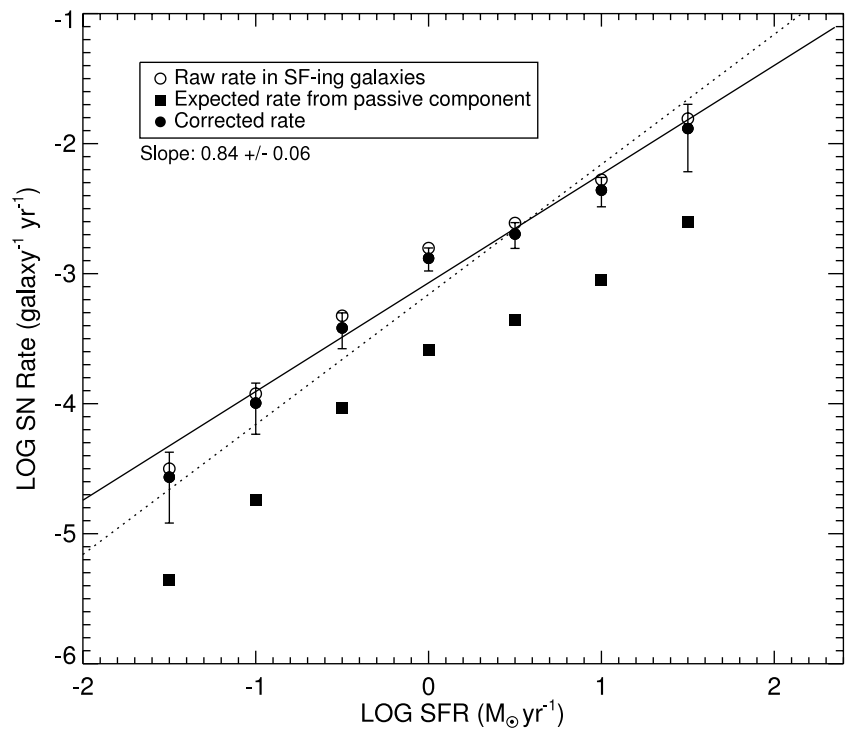

FIG. 9.- Number of SNe Ia per host galaxy as a function of host galaxy mean SFR measured over a 0.5 Gyr period (see text for details). The open circles show the raw rate in star-forming galaxies, with the squares showing the expected rate derived from the total stellar mass of field galaxies in each bin. The filled circles show the number per galaxy after the component from the stellar mass is removed. The solid line shows the best fit; the dotted line has a slope of unity. [See the electronic edition of the Journal for a color version of this figure.]

bin of mean SFR for this general galaxy population, we sum the total stellar mass in that bin and, using a value of $A$ determined from the passive galaxies in $\S 5.1$, calculate the number of SNe Ia expected from any old component. This old component is subtracted from each bin, leaving an excess of SNe Ia above that predicted from the stellar mass of the galaxies. We show this distribution in Figure 9. In this figure, we have combined the SNe Ia occurring in all types of star-forming galaxies rather than the two separate star-forming populations shown in Figure 8. As in $\S 5.1$, a Monte Carlo simulation is used to account for the uncertainties in the stellar mass and mean SFR estimates for the host galaxy population.

Figure 9 shows clear evidence for a component of $\mathrm{SNe}$ Ia beyond that dependent on the integrated stellar mass. The number of SNe Ia expected in each SFR bin from the stellar mass is smaller than actually observed; the majority of SNe Ia in starforming galaxies appear to arise from more recently formed stars than from old stars. Furthermore, the fraction of SNe Ia from the " $A$ " component appears fairly constant with the SFR of the galaxy, with a range of $14 \%-21 \%$. We also test whether the dependence on the mean SFR of a galaxy is linear by fitting the slope of the line, $n_{\mathrm{SFR}}$. The best fit is $n_{\mathrm{SFR}}=0.84 \pm 0.06$ with a $\chi^{2}=1.31$ (the slope is $n_{\mathrm{SFR}}=0.81 \pm 0.08$ for the spectroscopically confirmed $\mathrm{SNe}$ Ia). For comparison, a line with a slope of unity is also shown $\left(\chi^{2}=2.34\right)$. We estimate $B=4.1 \pm 0.7 \times$ $10^{-4} \mathrm{SNe} \mathrm{yr}^{-1}\left(M_{\odot} \mathrm{yr}^{-1}\right)^{-1}$ using this approach and enforcing a linear relationship.

\subsection{Bivariate Fits}

Although the last two sections provide a useful visualization of the SN Ia rate as a function of galaxy stellar mass and mean SFR, a more sophisticated bivariate technique that fits $A$ and $B$ simultaneously across all galaxy types will result in more accurate fit values, as the stellar masses and SFRs are partially correlated (Fig. 5). We assume the relationship is a linear function of only $A$ and $B$ (i.e., $n_{\text {mass }}$ and $n_{\mathrm{SFR}}$ are fixed) and perform a generalized linear least-squares fit in the galaxy mass/SFR plane.
This effectively fits the two components of the SN Ia rate simultaneously to the data. We perform this fit by converting Figure 5, the distribution of SN Ia host galaxies in the mass/SFR plane, into a probability of a SN Ia explosion in a given galaxy as a function of mass and SFR. The probability is calculated by binning the host distribution by galaxy mass and galaxy SFR, and dividing the number of SN hosts in each bin by the (incompleteness corrected) number of field galaxies similarly binned. This results in a twodimensional mass/SFR probability space that gives the likelihood of a SN Ia explosion in a galaxy as a function of both the galaxy stellar mass and mean SFR.

We fit two forms of equation (4) to this probability distribution: equation (4) with $A$ and $B$ both free, and the same function but with $B=0$, i.e., assuming the SN Ia rate depends only on host galaxy stellar mass (clearly the third alternative, fixing $A=0$, makes no sense, as plenty of SNe Ia explode in non-star-forming galaxies). The addition of the $B$ term as a free parameter $(B \neq 0)$ reduces the $\chi^{2}$ of the fit from 67 to 37 (60 degrees of freedom). Performing an $F$-test, we find that the null hypothesis that the extra $B$ term is not needed is rejected at $>99.99 \%$ probability. The fit results when fitting for both $A$ and $B$ with $n_{\text {mass }}=n_{\mathrm{SFR}}=1$ are $A=5.3 \pm 1.1 \times 10^{-14}\left(H_{0} / 70\right)^{2} \mathrm{SNe} \mathrm{y^{-1 }} M_{\odot}^{-1}$ and $B=3.9 \pm$ $0.7 \times 10^{-4}\left(H_{0} / 70\right)^{2} \mathrm{SNe} \mathrm{yr}^{-1}\left(M_{\odot} \mathrm{yr}^{-1}\right)^{-1}$ (these errors are statistical only). Using the formal covariance matrix of the fit, we calculate a correlation coefficient between the input masses and SFRs of -0.22 , indicating only a small correlation.

One important feature of Figure 9 is the slight nonlinear relationship between $\mathrm{SNR}_{\mathrm{Ia}}$ and SFR. Using the bivariate fit method, we can also derive best-fit values for $n_{\text {mass }}$ and $n_{\text {SFR }}$ by examining the $\chi^{2}$ variation of the best-fit as $n_{\text {mass }}$ and $n_{\mathrm{SFR}}$ are varied in the fitting function. We find minimum $\chi^{2}$ at $n_{\text {mass }}=1.00_{-0.10}^{+0.11}$ $n_{\mathrm{SFR}}=0.98_{-0.11}^{+0.12}$. These are consistent although more reliable than the estimates of $\S \S 5.1$ and 5.2, and are completely consistent with $n_{\text {mass }}=n_{\mathrm{SFR}}=1$.

Clearly, in an analysis such as these systematic errors are likely to be at least as large as the statistical ones quoted above. For example, using different IMFs (e.g., those of Salpeter [1955] or Rana \& Basu [1992]) can vary the fit values of $A$ and $B$ by around $10 \%-20 \%$ of their value compared to the IMF of Kroupa (2001); using different spectral libraries is likely to produce changes of a similar magnitude. Furthermore, although dust is included in the PÉGASE. 2 models, no dust extinction correction is made to individual galaxies, and systematic errors could clearly therefore be present in the SFR estimates, especially when considering the existing evidence for SFR-dependent extinction corrections (Hopkins et al. 2001; Sullivan et al. 2001). Hence, the values of $A$ and $B$ may change as more multiwavelength data become available for the host galaxies and better constrain the extinction properties of the galaxies.

\subsection{Comparison to Other Results}

The fits of $\oint 5.3$ show that the SN Ia rate in galaxies has a linear dependence on both stellar mass and younger stellar populations, which we parameterize via the galaxy SFR. We also derived parameters $A$ and $B$, which allow us to relate the mass and SFR of a galaxy to the probability of it hosting a SN Ia. We can compare our estimates of $A$ and $B$ with other similar numbers published in the literature.

Mannucci et al. (2005) calculated the low-redshift SN Ia rate per unit mass as a function of host galaxy morphological type from the SN sample of Cappellaro et al. (1999). In low-redshift E/S0 galaxies, they find a rate of $3.83_{-1.2}^{+1.4} \times 10^{-14} \mathrm{SNe} \mathrm{yr}^{-1} M_{\odot}^{-1}$ (in our cosmology), which agrees with our determination $(A=$ $5.3 \pm 1.1 \times 10^{-14}$ ) within the error bars. Given the different 
techniques for determining galaxy types (SED fitting vs. morphological typing) and the different search and efficiency calculations, this is an encouraging level of agreement. Our determination of $B\left[3.9 \pm 0.7 \times 10^{-4} \mathrm{SNe} \mathrm{yr}^{-1}\left(M_{\odot} \mathrm{yr}^{-1}\right)^{-1}\right]$ is, however, discrepant with values of SB05, who derived two different values, $10_{-5}^{+6}$ and $23_{-10}^{+10} \times 10^{-4}$ in our units. If we take the $\operatorname{SNLS} z=0.47$ SN Ia rate of $0.42 \times 10^{-4} h_{70}^{3} \mathrm{SNe} \mathrm{yr}^{-1} \mathrm{Mpc}^{-3}$ and assume that all of this rate is generated from the prompt component, with a star formation density at $z=0.47$ of $0.043 M_{\odot} \mathrm{yr}^{-1} \mathrm{Mpc}^{-3}$ (Hopkins $\&$ Beacom 2006), this implies an upper limit for $B$ of $B \lesssim 1.0 \times$ $10^{-3} \mathrm{SNe} \mathrm{yr}^{-1}\left(M_{\odot} \mathrm{yr}^{-1}\right)^{-1}$ at the lower end of the SB05 assumed values.

We can also estimate the predicted rate of SNe Ia in clusters from elliptical galaxies and compare to the observed cluster rate as a further consistency check. As instantaneous star formation in massive clusters can be suppressed relative to field galaxies (Couch et al. 2001), cluster SNe Ia are likely to be dominated by events from the old component. Gal-Yam et al. (2002) measured a cluster SN Ia rate of $0.392 h_{70}^{2} \mathrm{SNu} .{ }^{14}$ Assuming a typical elliptical mass-to-light ratio in $B$ band of 5-10, this converts to a SN Ia rate in clusters of 3.9-7.8 $\mathrm{SNe} \mathrm{yr}^{-1} M_{\odot}^{-1}$, in excellent agreement with our $A$-parameter determination.

We also check that the $A$ and $B$ values derived here are consistent with measured volumetric SN Ia rates. We use the cosmic SFH of Hopkins \& Beacom (2006), which gives the SFR of the universe as a function of redshift. Integrating this SFR gives the mass as a function of redshift, which in conjunction with our $A$ and $B$ values will predict the SN Ia rate. Care must be taken that a similar definition of mass is used here as is used when we derive $A$, i.e., the mass of stars that have died must be subtracted from the integral (see Fig. 7 for the difference this correction makes in the mass evolution of the cosmic SFH). To do this, we use the SFH of Hopkins \& Beacom (2006) as an input to PÉGASE.2 to calculate the total mass in stars as a function of redshift. We then use the $A$ and $B$ values from above to calculate the volumetric SN Ia rate (Fig. 10). The relative contributions of the two components evolves strongly with time. The young component provides $\sim 10 \%-20 \%$ of all SNe Ia at $z=0$, rising to $\sim 85 \%$ at $z=2$; the exact ratios depend strongly on the assumed cosmic SFH. Qualitatively similar trends were also predicted by Mannucci et al. (2006), based on completely different analysis and fitting techniques. This first fraction is quite low; we note that assuming that the likelihood of the old component decreases with age rather than remains constant, as in equation (4), would result in a larger fraction of SNe being generated from the prompt component at $z=0$, perhaps illustrating a limitation of the " $\mathrm{A}+\mathrm{B}$ " model.

The agreement with the volumetric rates is remarkably good, given the SFH used in the calculation (Hopkins \& Beacom 2006) is completely independent of the derivation of $A$ and $B$. All of the published rates are statistically consistent at the $2 \sigma$ level with the simple prompt + old model parameters derived here. The most deviant point is the $\simeq 1$ rate from Dahlen et al. (2004), but even this value differs from the model at only $1.8 \sigma$. The $A$ and $B$ model used here predicts a shallow redshift evolution of the SN Ia rate, one that does not evolve as fast as the cosmic SFH. Of course, the distribution of the two types will vary considerably with local galaxy density. In low-mass star-forming dwarf field galaxies, for example, the prompt component will be the source for essentially all SNe Ia. Yet, a substantial fraction of SNe Ia will always occur in $\mathrm{E} / \mathrm{S} 0$ galaxies (and perhaps the bulges of spiral

\footnotetext{
141 "SNu" is one SN per century per $10^{10}$ stellar $B$-band luminosities.
}

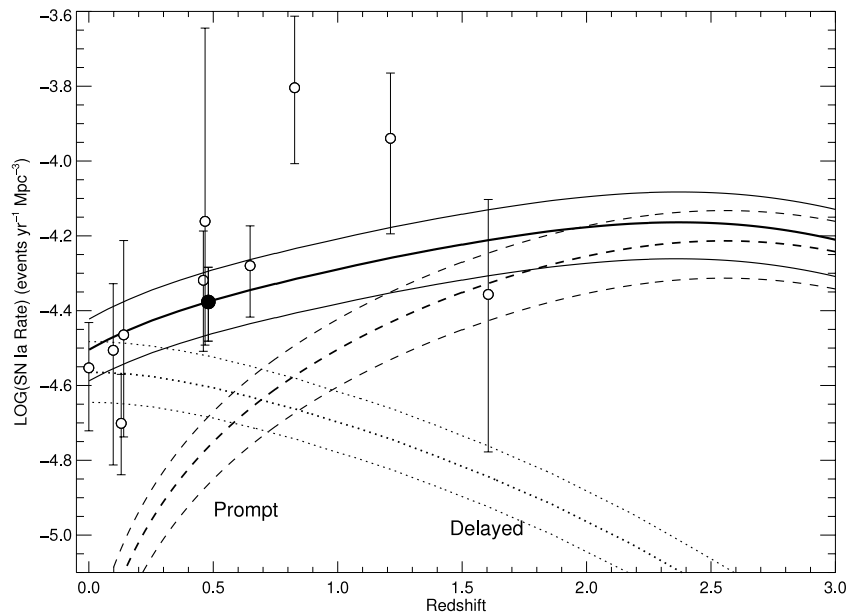

FIG. 10.-Predicted volumetric SN Ia rate as a function of redshift based on the $A$ and $B$ values from the bivariate fit of $\S 5$.3. The dotted lines denote the $A$ old component (plus limits from the statistical errors), and the dashed line denotes the $B$ prompt component. The solid line shows the sum of the two. The filled circle is the $z=0.47$ SNLS determination of the SN Ia rate of Neill et al. (2006). Open circles represent other SN Ia rate determinations from Cappellaro et al. (1999), Hardin et al. (2000), Pain et al. (2002), Tonry et al. (2003), Dahlen et al. (2004), and Blanc et al. (2004). We conservatively show the statistical and systematic error bars added in quadrature where both are given in these papers. The SFH of Hopkins \& Beacom (2006) is assumed.

galaxies) and in clusters due the large amount of old stellar mass locked up in these systems.

\section{SUPERNOVA PROPERTIES AS A FUNCTION OF STELLAR POPULATIONS}

The results of $\S 5$ show that the rate of SNe Ia in a given galaxy depends on both the evolved (old) stellar mass of that system and the young stellar content, parameterized via the SFR. Indeed, a simple mass scaling - assuming that the SN Ia rate is a simple function of galaxy stellar mass - is unable to reproduce the trends that we see. These results could have important implications for the use of SNe Ia as precise cosmological probes. At the very least, as discussed by SB05 and Mannucci et al. (2006), there must be a broad range of delay times between a binary system formation and $\mathrm{SN}$ explosion. Whether this is interpreted as being due to a single channel to a SN Ia (i.e., perhaps single degenerate or double degenerate) or via a two-channel path to a SN Ia (i.e., perhaps single degenerate and double degenerate), a distinct possibility is that the average properties of these prompt and old SN Ia populations could differ. In this section, we search for SN Ia properties that correlate with environment, or more particularly star formation activity (or the mean age of the stellar population) in their host galaxies.

One key observable affecting the utility of SNe Ia as cosmological probes is the light-curve shape/luminosity relationship (Phillips 1993), which corrects the luminosity of SNe Ia according to the width of their light curves - the so-called "brighterslower" relationship. In this paper, we parameterize the SN Ia light curves using the stretch parameter $s$ (e.g., Perlmutter et al. 1997), which linearly stretches or contracts the time axis of a template SN Ia light curve around the time of maximum light to best fit the observed light curve of the SN being fit. At low redshift, a trend of SN Ia absolute luminosity —or equivalently, light-curve width cast in terms of $\Delta m_{15}(B)$, the decline in $B$-band magnitudes 15 days after maximum light - versus galaxy morphological type has been observed (Hamuy et al. 1995, 1996, 2000; Riess et al. 1999; see Gallagher et al. [2005] for a recent 

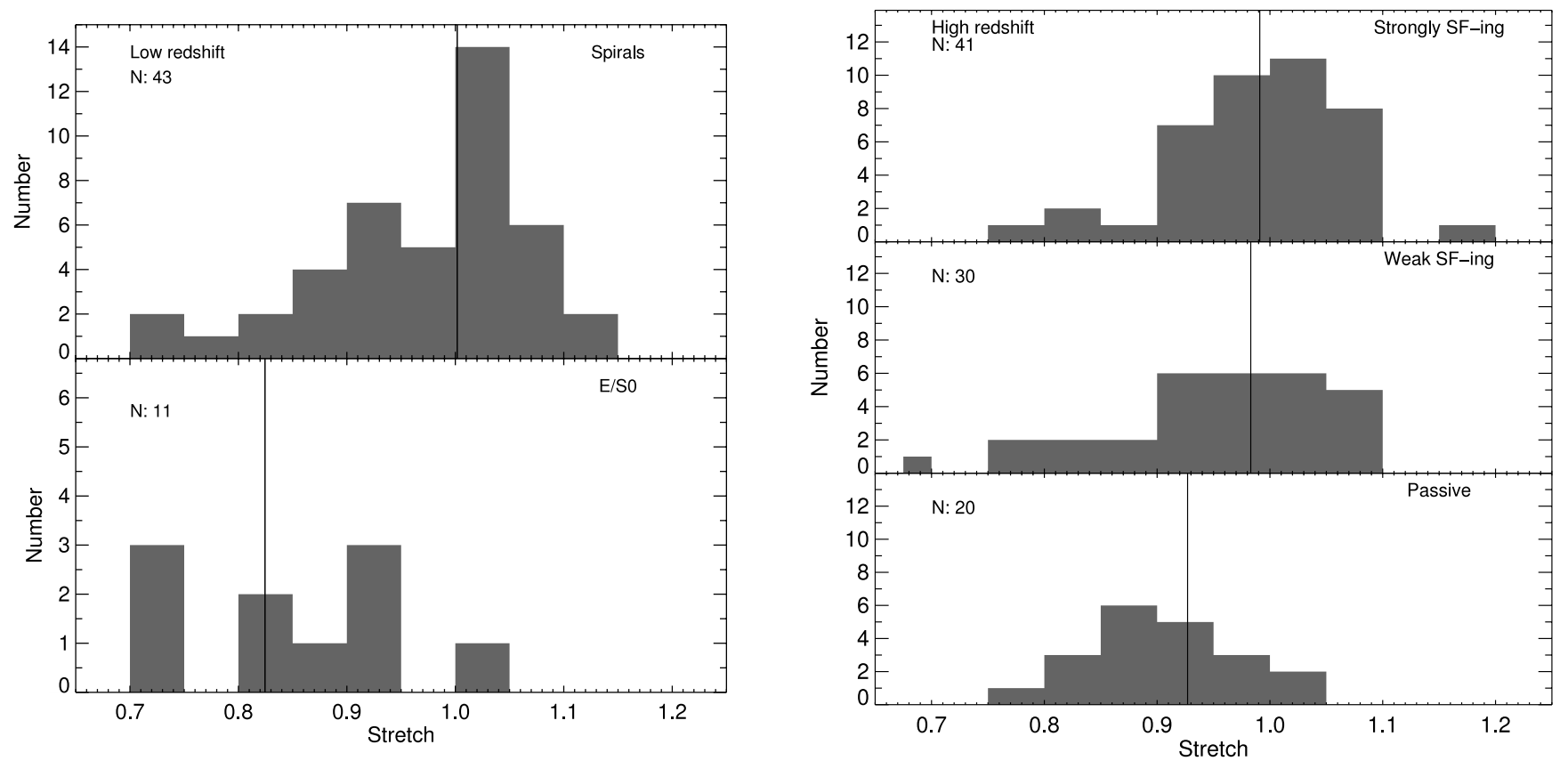

FIG. 11.-Distribution of the SN Ia light-curve shape parameter stretch, separated according to the specific SFR of the host galaxy. The left panel shows low-redshift SNe Ia; the right panel shows high-redshift SNLS SNe Ia. The typical precision on the stretch measure is $\pm 0.01-0.02$, i.e., smaller than the bin width of the histograms. Left: The top panel shows galaxies morphologically classified as spirals; the bottom panel shows those $\mathrm{SNe}$ in elliptical or S0 galaxies. Right: The top panel shows galaxies with a specific SFR $(\mathrm{sSFR})$ of $\log (\mathrm{sSFR})>-9.5$, the middle panel shows those with $-12.0 \leq \log (\mathrm{sSFR}) \leq-9.5$, and the bottom panel shows those with $\log (\mathrm{sSFR})<-12.0$. The vertical lines show the positions of the median stretch in each histogram. [See the electronic edition of the Journal for a color version of this figure.]

compilation). These authors show that brighter SNe Ia [or more precisely, high-stretch/low- $\left.\Delta m_{15}(B)\right]$ are preferentially located in late-type (younger) galaxies.

Here, we compare the low-redshift distribution to that seen at high redshift in the current SNLS sample (Fig. 11). We use the specific SFR classification of the SNLS host galaxies at high redshift and take classifications for the low-redshift SNe hosts from either the literature (Riess et al. 1999; Hamuy et al. 2000; van den Bergh et al. 2002) or the NASA IPAC Extragalactic Database (NED), simplifying onto an elliptical (E/S0), earlytype ( $\mathrm{Sa}-\mathrm{Sbc})$, and late-type ( $\mathrm{Sc}$ and later) classification scheme. We refit the published light curves for the low-redshift SNe Ia with the same method as the high-redshift $\mathrm{SNe}$ Ia to ensure a consistent definition of "stretch" between low and high redshift.

The trend in Figure 11 across galaxy type is clear-passive galaxies with a small (or zero) specific SFR tend to host lower stretch $\mathrm{SNe}$. Although the low- $z$ trend was already well known, this is the first time the effect has been seen at high $z$ (e.g., Sullivan et al. 2003), presumably because the SNLS probes a wider range in stretch than previous high- $z$ surveys, particularly at lower stretches (fainter SNe). Kolmogorov-Smirnov (K-S) tests can be used to give some statistical guide as to whether these various stretch distributions are similar; these are summarized in Table 4 . We show that at high redshift, the stretch distributions between $\mathrm{SNe}$ in passive galaxies versus those in both types of star-forming galaxies are different with $>98 \%$ confidence. We also show that the stretch distributions for spiral galaxies at low $z$ and star-forming galaxies at high $z$ are essentially identical.

The distributions in elliptical galaxies at low- $z$ versus passive galaxies at high- $z$ are formally different at the $90 \%$ level, although this is caused entirely by the lack of very low stretch high- $z \mathrm{SNe}$ $(s \sim 0.7)$. One possibility is that the delay times for the lowest stretch SNe Ia are long enough to preclude their existence at high redshift (Howell 2001). Another is that as these $\mathrm{SNe}$ are the fainter subsample, this absence could be attributed to selection effects.
In general, selection effects are unlikely to be responsible for the other trends that we observe. The main trend concerns an absence of high-stretch (i.e., brighter) SNe Ia in passive systems at high redshift; a selection or Malmquist bias in passive systems would lead to the opposite trend, i.e., a decrease in the lower stretch (fainter) SNe Ia in these systems. Furthermore, the trends remain unchanged if the sample is culled at $z=0.6$ (where Malmquist effects will be smaller) rather than at $z=0.75$.

These trends seem highly suggestive of differing properties between prompt and older SNe Ia. The passive galaxies, with a $\mathrm{SFR}=0$ and an average older stellar population, host events with average stretches differing from those prompt SNe Ia found in galaxies comprised of younger stellar populations. Of course, galaxies with an average young stellar population are still likely to host low-stretch SNe Ia due to the older stellar populations that must be present in all but the youngest starburst galaxies, although the reverse is less likely to be true. We can attempt to identify this low-stretch population in Figure 11 using a simple mass scaling

TABLE 4

K-S Tests on the Stretch Distributions at Low and High Redshift Probability That Distribution is the Same

K-S Test Sample (\%)

Low $-z$ E/S0 $\rightarrow$ high- $z$ passive .....................

Low- $z$ spirals $\rightarrow$ high $z$ SFR $>0$......

High- $z$ passive $\rightarrow$ high- $z$ weak ${ }^{\mathrm{a}} \mathrm{SF}^{\mathrm{b}}$. 10

High- $z$ passive $\rightarrow$ high- $z$ strong $\mathrm{SF}^{b}$

95

High- $z$ passive $\rightarrow$ high- $z$ all $\mathrm{SF}^{\mathrm{b}}$...

High $z$ weak SF $\rightarrow$ high $z$ strong SF $^{\mathrm{b}}$

\footnotetext{
a The weak and strong star-forming galaxies are divided based on their specific SFR; see $\S 5$.

b $\mathrm{SF}=$ star-forming.
} 


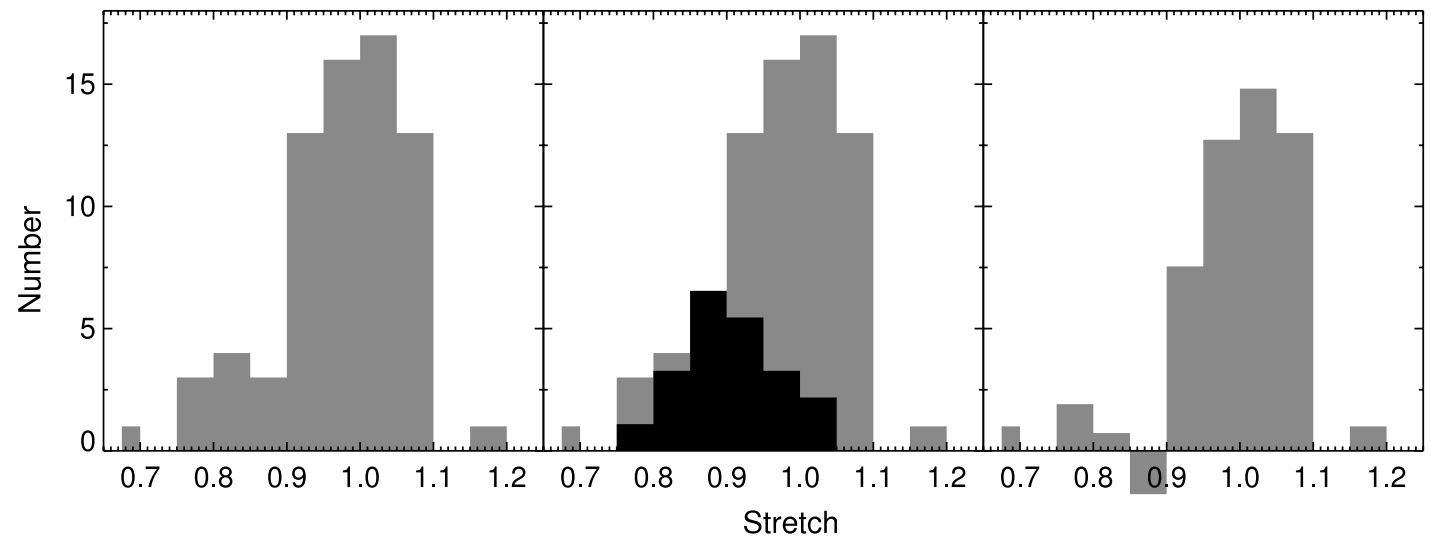

FIG. 12.-Effect of subtracting the SN Ia stretch distribution in passive galaxies from the distribution in star-forming galaxies. The left panel shows the distribution in all star-forming galaxies; the middle panel shows the passive galaxy distribution overplotted scaled by the ratio of the total mass in passive galaxies to the total mass in star formers. The right panel shows the remaining distribution after subtracting the scaled passive galaxy distribution. [See the electronic edition of the Journal for a color version of this figure.]

technique in the context of the single-degenerate progenitor system scenario.

We sum the total stellar mass in all the field passive galaxies and in all the star-forming galaxies. We then scale the stretch distribution in the old galaxies by the ratio of these two total masses and overplot on the stretch distributions of SNe Ia in the younger hosts. Subtracting this scaled population leaves just the residual stretch distribution remaining (Fig. 12). Clearly, other ratios could be used to scale the passive distribution prior to subtraction. We found that the residual stretch distribution was similar when using various different mass ranges (e.g., $0.8-3.0 M_{\odot}$ ) to calculate the scaling ratios.

The resulting subtraction is remarkably clean given the simple assumption that was made when scaling the low-stretch SN distribution. The low-stretch distribution in passive galaxies seems able to reproduce the distribution of low-stretch SNe Ia in starforming galaxies, within the statistical errors. This seems to provide supporting evidence that not only is age a key parameter driving the SN Ia rate (see $\S 5$ ), but that it also provides a physical parameter partially controlling the observed SN to SN stretch variation.

\section{CONCLUSIONS}

In this paper, we have examined the rates and properties of high-redshift Type Ia supernovae (SNe Ia) discovered via the Supernova Legacy Survey (SNLS) as a function of the stellar mass and star formation in their host galaxies. Our principal findings are as follows.

1. The SN Ia rate per unit stellar mass is a strong function of host galaxy specific SFR. More strongly star-forming (later type) galaxies host around 10 times as many SNe Ia per unit mass than do passive galaxies with a zero SFR, similar to trends observed in the local universe by Mannucci et al. (2005).

2. The number of SNe Ia per galaxy in passive galaxies closely tracks the stellar mass of the host system with a linear relationship. Although a relationship between the number of SNe per galaxy and galaxy mass is also seen in star-forming galaxies, it is not a simple linear dependence $(n=\simeq 0.7 \pm 0.08)$, with an excess of $\mathrm{SNe}$ Ia in low-mass star-forming galaxies when compared to that in non-star-forming galaxies.

3. We find a clear relationship between the number of SNe Ia per galaxy and the galaxy mean SFR averaged over the last $0.5 \mathrm{Gyr}$, even after removing any signal derived from the $\mathrm{SN} /$ host mass relationship found above. More strongly star-forming systems host more SNe Ia per galaxy than lower SFR systems, with a best-fitting slope of $n_{\mathrm{SFR}}=0.98_{-0.11}^{+0.12}$.

4. By approximating the SN Ia rate as a bivariate linear function of host galaxy stellar mass $(M)$ and host galaxy mean SFR averaged over the last $0.5 \mathrm{Gyr}\left(\dot{M}_{\text {new }}\right)$, we find that the SN Ia rate in a galaxy is well represented by $\operatorname{SNR}_{\mathrm{Ia}}(t)=A M(t)+B \dot{M}_{\text {new }}(t)$, with $A=5.3 \pm 1.1 \times 10^{-14}\left(H_{0} / 70\right)^{2} \mathrm{SNe} \mathrm{yr}^{-1} M_{\odot}^{-1}$ and $B=$ $3.9 \pm 0.7 \times 10^{-4}\left(H_{0} / 70\right)^{2} \mathrm{SNe} \mathrm{yr}^{-1}\left(M_{\odot} \mathrm{yr}^{-1}\right)^{-1}$.

5. We demonstrate, for the first time at high redshift, a relationship between star formation in a host galaxy and the SN light-curve width (stretch). We find that high-stretch (brighter) SNe Ia are exclusively hosted by star-forming galaxies, while non-star-forming galaxies only host low-stretch SNe Ia. We show that the SN stretch distributions in low-redshift and high-redshift spiral galaxies are statistically identical at $95 \%$ confidence, with the distribution in elliptical galaxies identical at low and high redshift except for the lowest stretch SNe Ia, which are not present at high redshift.

6. We show that the total SN Ia stretch distribution in highredshift star-forming galaxies can be well represented by a combination of a high-stretch component and a low-stretch component equivalent to the low-stretch distribution in passive galaxies, scaled by the ratio of the total mass in passive galaxies to that in star-forming galaxies. The indication is that not only can $\mathrm{SNe}$ Ia be generated from both old and young progenitor systems, but there is a systematic difference in the mean light-curve properties of the two components.

These conclusions could have implications for the use of SNe Ia to determine cosmological parameters. As the cosmic SFR density shows sharp evolution as a function of redshift, the relative mix of the two SN Ia components will change correspondingly (see Fig. 10), assuming the efficiency of generating a SN I from a given progenitor scenario is invariant with redshift. The prompt component should supply the dominant fraction of observed SNe Ia at high redshift, with the old component producing a larger fraction at low redshift. The crossover point, where the contributions from the two components is the same, is around $z \sim 0.5-0.9$ assuming the Hopkins \& Beacom (2006) SFH.

There are at least two direct implications for the use of SNe Ia as cosmological probes. The first is that $\mathrm{SNe}$ Ia should be found even at very high redshift $(z \sim 3-4$ and above). While SN Ia 
models with significant delay times would preclude the existence of SN Ia when the universe was so young $(t \simeq 1-2 \mathrm{Gyr})$, the delay time for the prompt component of SNe Ia is very short. The implication is that SNe Ia could therefore be used as cosmological probes up until the highest redshifts at which stars are being formed. SNe Ia at $z=4-5$ would be within easy range of future facilities such as the James Webb Space Telescope and the various proposed 30-100 m class ground-based telescopes.

The second implication is that an excellent understanding of the light-curve shape/luminosity correction in different environments will be essential to fully exploit $\mathrm{SNe}$ Ia in measurements of $\langle w\rangle$ and, in particular, for the more sensitive task of measuring any variation of $w$ with redshift. The study and classification of SN Ia environment, using, for example, similar techniques to those presented here may become as important a part of determining future cosmological constraints as measuring the light curves of the SNe Ia themselves. Surveys that routinely obtain detailed information on the environment of all confirmed $\mathrm{SNe}$ detected will be ideally placed to perform studies of this nature.

The SNLS collaboration gratefully acknowledges the assistance of Pierre Martin and the CFHT Queued Service Observa- tions team. Jean-Charles Cuillandre and Kanoa Withington were also indispensable in making possible real-time data reduction at CFHT. We thank Lars Bildsten and Evan Scannapieco for useful discussions, and Andrew Hopkins for providing a copy of Hopkins \& Beacom (2006) prior to submission. Based on observations obtained with MegaPrime/MegaCam, a joint project of CFHT and CEA/DAPNIA, at the Canada-France-Hawaii Telescope (CFHT), which is operated by the National Research Council (NRC) of Canada, the Institut National des Sciences de l'Univers of the Centre National de la Recherche Scientifique (CNRS) of France, and the University of Hawaii. This work is based in part on data products produced at the Canadian Astronomy Data Centre as part of the CFHT Legacy Survey, a collaborative project of NRC and CNRS. Canadian collaboration members acknowledge support from NSERC and CIAR; French collaboration members from CNRS/IN2P3, CNRS/INSU, and CEA. This research has made use of the NASA/IPAC Extragalactic Database (NED), which is operated by the Jet Propulsion Laboratory, California Institute of Technology, under contract with the National Aeronautics and Space Administration. The views expressed in this article are those of the authors and do not reflect the official policy or position of the United States Air Force, Department of Defense, or the US government.

Facilities: CFHT, Gemini, Keck, VLT.
Astier, P., et al. 2006, A\&A, 447, 31

Barris, B. J., \& Tonry, J. L. 2006, ApJ, 637, 427

Benítez, N., et al. 2004, ApJS, 150, 1

Bernstein, R. A., Freedman, W. L., \& Madore, B. F. 2002, ApJ, 571, 56

Bertin, E., \& Arnouts, S. 1996, A\&AS, 117, 393

Blanc, G., et al. 2004, A\&A, 423, 881

Bolzonella, M., Miralles, J.-M., \& Pelló, R. 2000, A\&A, 363, 476

Boulade, O., et al. 2003, Proc. SPIE, 4841, 72

Brinchmann, J., Charlot, S., White, S. D. M., Tremonti, C., Kauffmann, G., Heckman, T., \& Brinkmann, J. 2004, MNRAS, 351, 1151

Brinchmann, J., \& Ellis, R. S. 2000, ApJ, 536, L77

Bundy, K., Ellis, R. S., \& Conselice, C. J. 2005, ApJ, 625, 621

Buzzoni, A. 2002, AJ, 123, 1188 2005, MNRAS, 361, 725

Calzetti, D., Armus, L., Bohlin, R. C., Kinney, A. L., Koornneef, J., \& StorchiBergmann, T. 2000, ApJ, 533, 682

Cappellaro, E., Evans, R., \& Turatto, M. 1999, A\&A, 351, 459

Coleman, G. D., Wu, C., \& Weedman, D. W. 1980, ApJS, 43, 393

Couch, W. J., Balogh, M. L., Bower, R. G., Smail, I., Glazebrook, K., \& Taylor, M. 2001, ApJ, 549, 820

Dahlen, T., et al. 2004, ApJ, 613, 189

Della Valle, M., \& Livio, M. 1994, ApJ, 423, L31

Della Valle, M., Panagia, N., Padovani, P., Cappellaro, E., Mannucci, F., \& Turatto, M. 2005, ApJ, 629, 750

Donas, J., Deharveng, J. M., Laget, M., Milliard, B., \& Huguenin, D. 1987, A\&A, 180,12

Felten, J. E. 1976, ApJ, 207, 700

Fioc, M., \& Rocca-Volmerange, B. 1997, A\&A, 326, 950

Gallagher, J. S., Garnavich, P. M., Berlind, P., Challis, P., Jha, S., \& Kirshner, R. P. 2005, ApJ, 634, 210

Gal-Yam, A., \& Maoz, D. 2004, MNRAS, 347, 942

Gal-Yam, A., Maoz, D., \& Sharon, K. 2002, MNRAS, 332, 37

Glazebrook, K., et al. 2004, Nature, 430, 181

Graham, A. W., \& Driver, S. P. 2005, Publ. Astron. Soc. Australia, 22, 118

Grazian, A., et al. 2006, A\&A, 449, 951

Greggio, L. 2005, A\&A, 441, 1055

Guzman, R., et al. 1997, ApJ, 489, 559

Gwyn, S. D. J., \& Hartwick, F. D. A. 1996, ApJ, 468, L77

Hamuy, M., Phillips, M. M., Maza, J., Suntzeff, N. B., Schommer, R. A., \& Aviles, R. 1995, AJ, 109, 1

Hamuy, M., Phillips, M. M., Suntzeff, N. B., Schommer, R. A., Maza, J., \& Aviles, R. 1996, AJ, 112, 2398

Hamuy, M., Trager, S. C., Pinto, P. A., Phillips, M. M., Schommer, R. A., Ivanov, V., \& Suntzeff, N. B. 2000, AJ, 120, 1479

Hardin, D., et al. 2000, A\&A, 362, 419

\section{REFERENCES}

Hillebrandt, W., \& Niemeyer, J. C. 2000, ARA\&A, 38, 191

Holwerda, B. W. 2005, preprint (astro-ph/0512139)

Hopkins, A. M., \& Beacom, J. F. 2006, ApJ, in press (astro-ph/0601463)

Hopkins, A. M., Connolly, A. J., Haarsma, D. B., \& Cram, L. E. 2001, AJ, 122, 288

Howell, D. A. 2001, ApJ, 554, L193

Howell, D. A., et al. 2005, ApJ, 634, 1190

Ilbert, O., et al. 2005, A\&A, 439, 863

Infante, L. 1987, A\&A, 183, 177

Kennicutt, R. C. 1998, ARA\&A, 36, 189

Kinney, A. L., Calzetti, D., Bohlin, R. C., McQuade, K., Storchi-Bergmann, T., \& Schmitt, H. R. 1996, ApJ, 467, 38

Kron, R. G. 1980, ApJS, 43, 305

Kroupa, P. 2001, MNRAS, 322, 231

2002, Science, 295, 82

Le Borgne, D., \& Rocca-Volmerange, B. 2002, A\&A, 386, 446

Le Borgne, D., Rocca-Volmerange, B., Prugniel, P., Lançon, A., Fioc, M., \& Soubiran, C. 2004, A\&A, 425, 881

Madau, P., Della Valle, M., \& Panagia, N. 1998a, MNRAS, 297, L17

Madau, P., Pozzetti, L., \& Dickinson, M. 1998b, ApJ, 498, 106

Magnier, E. A., \& Cuillandre, J.-C. 2004, PASP, 116, 449

Mannucci, F., Della Valle, M., \& Panagia, N. 2006, MNRAS, 370, 773

Mannucci, F., Della Valle, M., Panagia, N., Cappellaro, E., Cresci, G., Maiolino, R.,

Petrosian, A., \& Turatto, M. 2005, A\&A, 433, 807

Maoz, D., \& Gal-Yam, A. 2004, MNRAS, 347, 951

McCarthy, P. J., et al. 2004, ApJ, 614, L9

Menanteau, F., Abraham, R. G., \& Ellis, R. S. 2001, MNRAS, 322, 1

Neill, D., et al. 2006, AJ, 132, 1126

Oemler, A., \& Tinsley, B. M. 1979, AJ, 84, 985

Oke, J. B., \& Gunn, J. E. 1983, ApJ, 266, 713

Pain, R., et al. 2002, ApJ, 577, 120

Perlmutter, S., et al. 1997, ApJ, 483, 565 1999, ApJ, 517, 565

Phillips, M. M. 1993, ApJ, 413, L105

Rana, N. C., \& Basu, S. 1992, A\&A, 265, 499

Riess, A. G., et al. 1998, AJ, 116, 1009 1999, AJ, 117, 707

Salpeter, E. E. 1955, ApJ, 121, 161

Scannapieco, E., \& Bildsten, L. 2005, ApJ, 629, L85 (SB05)

Schmidt, M. 1968, ApJ, 151, 393

Smith, J. A., et al. 2002, AJ, 123, 2121

Strolger, L.-G., et al. 2004, ApJ, 613, 200 2005, ApJ, 635, 1370

Sullivan, M., Mobasher, B., Chan, B., Cram, L., Ellis, R., Treyer, M., \& Hopkins, A. 2001, ApJ, 558, 72 
Sullivan, M., et al. 2003, MNRAS, 340, 1057 2006, AJ, 131, 960

Tonry, J. L., et al. 2003, ApJ, 594, 1

Treu, T., et al. 2005, ApJ, 633, 174

van den Bergh, S. 1990, PASP, 102, 1318 van den Bergh, S., Li, W., \& Filippenko, A. V. 2002, PASP, 114, 820

Wang, L., Höflich, P., \& Wheeler, J. C. 1997, ApJ, 483, L29

Willmer, C. N. A., et al. 2006, ApJ, 647, 853

Yi, S. K., et al. 2005, ApJ, 619, L111 